\title{
Diagnostic imaging guide for autoimmune pancreatitis
}

\author{
Masaaki Takahashi ${ }^{1} \cdot$ Yasunari Fujinaga $^{1} \cdot$ Kenji Notohara $^{2} \cdot$ Takashi Koyama $^{3} \cdot$ Dai Inoue $^{4} \cdot$ Hiroyuki Irie $^{5}$. \\ Toshifumi Gabata ${ }^{4} \cdot$ Masumi Kadoya $^{6}$. Shigeyuki Kawa ${ }^{7} \cdot$ Kazuichi Okazaki $^{8} \cdot$ on behalf of the Working Group \\ Members of The Research Program on Intractable Diseases from the Ministry of Labor, Welfare of Japan
}

Received: 26 March 2020 / Accepted: 1 April 2020 / Published online: 15 April 2020

(C) Japan Radiological Society 2020

\begin{abstract}
The International Consensus Diagnosis Criteria for autoimmune pancreatitis (AIP) has been published internationally for the diagnosis of AIP. However, since the revisions in 2006 and 2011, the Clinical Diagnostic Criteria for Autoimmune Pancreatitis 2018 have been published. The criteria were revised based the Clinical Diagnostic Criteria 2011, and included descriptions of characteristic imaging findings such as (1) pancreatic enlargement and (2) distinctive narrowing of the main pancreatic duct. In addition, pancreatic duct images obtained by magnetic resonance cholangiopancreatography as well as conventional endoscopic retrograde pancreatography were newly adopted. The guideline explains some characteristic imaging findings, but does not contain descriptions of the imaging methods, such as detailed imaging parameters and optimal timings of dynamic contrast-enhanced computed tomography/magnetic resonance imaging. It is a matter of concern that imaging methods can vary from hospital to hospital. Although other characteristic findings have been reported, these findings were not described in the guideline. The present paper describes the imaging methods for obtaining optimal images and the characteristic imaging findings with the aim of standardizing image quality and improving diagnostic accuracy when radiologists diagnose AIP in actual clinical settings.
\end{abstract}

Keywords Autoimmune pancreatitis $\cdot$ IgG4-related disease $\cdot$ Computed tomography $\cdot$ Magnetic resonance imaging . Diagnostic imaging guide

Yasunari Fujinaga

fujinaga@shinshu-u.ac.jp

1 Department of Radiology, Shinshu University School of Medicine, 3-1-1 Asahi, Matsumoto, Nagano 390-8621, Japan

2 Department of Anatomic Pathology, Kurashiki Central Hospital, Kurashiki, Okayama, Japan

3 Department of Diagnostic Radiology, Kurashiki Central Hospital, Kurashiki, Okayama, Japan

4 Department of Radiology, Kanazawa University Graduate School of Medical Science, Kanazawa, Japan

5 Department of Radiology, Faculty of Medicine, Saga University, Saga, Japan

6 Department of Radiology, Hohseikai Marunouchi Hospital, Matsumoto, Japan

7 Department of Internal Medicine, Matsumoto Dental University, Shiojiri, Japan

8 Department of Gastroenterology and Hepatology, Kansai Medical University, Osaka, Japan

\section{Introduction}

Regarding imaging findings for autoimmune pancreatitis (AIP), pancreatic swelling (diffuse and focal) and irregular main pancreatic duct (MPD) narrowing on endoscopic retrograde pancreatography (ERP) or magnetic resonance cholangiopancreatography (MRCP) were included in the Japanese Clinical Diagnostic Criteria for Autoimmune Pancreatitis 2018 (JPS2018) [1]. However, it is often difficult to judge these findings because their descriptions in the guidelines are insufficient. In addition, there are other characteristic findings that are useful for diagnosis of AIP.

In the JPS2018, pancreatic duct evaluation by MRCP alone is permitted because the quality of MRCP has improved and useful MRCP findings for diagnosing AIP were recently reported [2-4]. This also takes into account the fact that invasive ERP is no longer being performed in recent years. However, there is a problem in AIP diagnosis with equivalent accuracy among individual radiologists. There are no descriptions about the imaging methods, 
such as detailed imaging parameters and optimal timings of dynamic contrast-enhanced computed tomography (CT)/ magnetic resonance imaging (MRI), in the JPS2018 or in the 2013 amendment of the Japanese Consensus Guidelines for AIP [1, 5-7]. It is a fact that the imaging methods used in different hospitals can differ from one another. Because the JPS2018 is not sufficient to enable all radiologists to achieve accurate diagnosis of AIP, there is a need for further guidance to make this possible and for easy-to-understand manuals that can be compared with the diagnostic images of AIP patients.

We aimed to standardize the image examination methods and improve the diagnostic accuracy so that radiologists can accurately diagnose AIP. Optimal imaging methods to obtain good diagnostic images that cannot be covered by the guidelines and characteristic radiologic findings were described in a comprehensive way, together with radiologic images of AIP patients.

In this guidance, "AIP" means type 1 AIP, if there is no other particular explanation.

\subsection{Recommended imaging methods}

In many cases, AIP cannot be diagnosed accurately using only a single modality. It is desirable that clinicians and radiologists understand this and make a comprehensive diagnosis using multiple imaging modalities with adequate information sharing.

To avoid artifacts and effects of inflammation, radiologic images should be obtained before endoscopic retrograde biliary drainage (ERBD) and stenting in the bile duct.

\subsubsection{Description}

Comprehensive diagnosis using multiple modalities is mandatory for accurate diagnosis of AIP, and ultrasound (US) including abdominal US and endoscopic ultrasonography (EUS), CT including non-contrast $\mathrm{CT}$ and contrastenhanced CT, nuclear medicine including fluorodeoxyglucose-positron-emission tomography (FDG-PET) and Ga-67 scintigraphy, and ERP are recommended. Although EUSguided biopsy is another important method, it has not been described in this article.

\subsection{Important points for CT examination}

Optimal imaging methods and scan timings for dynamic contrast-enhanced CT (DCE-CT) are important for accurate assessment of pancreatic findings.

Whenever possible, DCE-CT should be performed.

The scan range should be set with consideration of the detection of extrapancreatic lesions.

\subsubsection{Descriptions}

Multi-row detector CT ( $\geq 64$ rows) is recommended. To reduce artifacts, it is recommended that the $\mathrm{CT}$ scans are performed with elevation of the arm. It is also recommended that the scan range is set with consideration of the detection of extrapancreatic lesions. Specifically, it is preferable that the cranial margin includes the lacrimal grands and the caudal margin includes the prostatic glands. Meanwhile, radiation exposure needs to be considered. Chest CT is obtained at full inspiration and abdominal-pelvic $\mathrm{CT}$ is obtained at full expiration.

For detailed assessment of the pancreatic findings, the slice thickness is $\leq 3 \mathrm{~mm}$ and thin-slice images are reconstructed with a focus on the pancreatic lesion if necessary. DCE-CT is performed using a non-ionic water-soluble iodine-containing contrast agent $(300-370 \mathrm{mgI})$ at a rate of $2-3 \mathrm{ml} / \mathrm{s}$ (recommended $\geq 3 \mathrm{ml} / \mathrm{s}$ ) via a catheter $(20-22$ $\mathrm{G})$ placed in the cubital vein. The total dose of the contrast agent is recommended to be $\geq 2 \mathrm{ml} / \mathrm{kg}$ body weight. DCE$\mathrm{CT}$ is obtained in multiple phases including the pancreatic phase (35-45 s after injection start) and delayed phase (130 s after injection start) [8]. A bolus tracking method is also recommended to obtain the optimal scan timing. Dual-energy CT may be useful for reducing the dose of the contrast agent and improving the contrast effect.

\subsection{Important points for MRI examination}

Multidirectional and appropriate image acquisition including MRCP and dynamic contrast-enhanced MRI (DCE-MRI) is necessary for accurate assessment of a pancreatic lesion. Whenever possible, DCE-MRI should be performed. Measures are necessary to reduce artifacts.

\subsubsection{Description}

Compared with CT, MRI provides better tissue contrast and plays an important role in the diagnosis of AIP. However, MRI has disadvantages, including differences in MR units and scan parameters among individual hospitals and longer acquisition times. Therefore, it is necessary to acquire MR images according to the circumstances within each hospital after discussions with clinicians and technicians.

It is recommended that the static magnetic field of the MR unit is $\geq 1.5 \mathrm{~T}$ ( $3 \mathrm{~T}$ is preferable). The scan range should include the whole pancreas. The recommended protocols for pancreatic MRI are as follows: ultrafast T2-weighted spin-echo imaging (UF-T2WI) reconstructed with partial Fourier imaging (axial and coronal), fat-suppressed T2-weighted spin-echo imaging (FS-T2WI) (axial), 2D or 3D T1-weighted gradient-echo imaging (GRE) including inphase, opposed-phase, and fat-suppression imaging (axial), 
diffusion-weighted imaging (DWI) including two or more $b$ values (axial), DCE-MRI using 3D GRE (axial), and MRCP using 3D T2WI (variable flip angle method). It is preferable that the slice thickness for UF-T2WI and DCE-MRI is $\leq 4 \mathrm{~mm}$, while that for the other sequences is $\leq 5 \mathrm{~mm}$. Axial-oblique or coronal-oblique images are sometimes useful because the whole pancreas can be observed in a single section on such images (Fig. 1).

On UF-T2WI, the association between the lesion and the MPD is demonstrated well. If the image quality on MRCP is degraded by artifacts, UF-T2WI can compensate for MRCP. The lesion-to-pancreas contrast is better on FS-T2WI than on T2WI without fat suppression. Because of the long acquisition time, FS-T2WI and DWI are usually obtained using a respiratory gating technique. On fat-suppressed T1-weighted imaging (FS-T1WI), the signal intensity of the pancreatic parenchyma is usually higher than that of the liver parenchyma. Therefore, FS-T1WI is useful for detection of a pancreatic lesion.

MRCP, including MR hydrography, is a kind of T2WI with a tenfold longer echo time than T2WI. If the breathing is unstable, the image quality on MRCP will be poor even when a respiratory gating technique is used. In such cases, additional acquisition of thick-slab 2D MRCP with breathholding is recommended. Recently, a compressed sensing technique that enables acquisition of 3D MRCP with breathholding was developed. The image quality on MRCP may be improved with an oral contrast agent that suppresses the water signals in the digestive tract. MRCP is a non-invasive technique that can visualize the pancreatic duct without a contrast agent. However, its image resolution is lower than that of ERP. It should be noted that MRCP directly depicts the pancreatic duct in vivo and its findings sometimes differ from those of ERP because of the contrast agent injection in ERP and the partial volume effect in MRCP $[9,10]$ (Fig. 2). If the MPD is obstructed, the upstream of the MPD

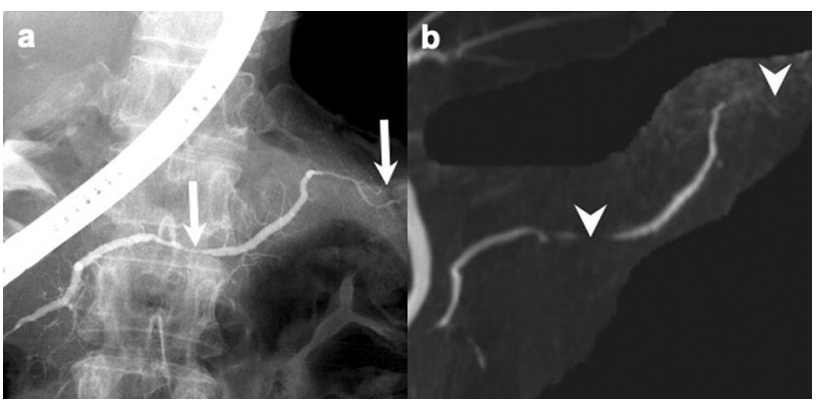

Fig. 2 Difference in depiction of the main pancreatic duct (MPD) and branch between magnetic resonance cholangiopancreatography (MRCP) and endoscopic retrograde pancreatography (ERP). a An ERP image shows MPD narrowing in the pancreatic body and tail (arrows). b On an MRCP image, MPD narrowing is seen as invisible areas (arrowheads), and the depiction of the branch duct is poor

is not demonstrated on ERP. In such cases, MRCP has an advantage because MRCP can visualize this region (Fig. 3). On MRCP, it is usually difficult to visualize the branch of the pancreatic duct because of the lower spatial resolution compared with ERP. However, a high-quality image of the pancreatic duct can be obtained using the partial maximum intensity projection (MIP) [11] (Fig. 4).

It is recommended that DCE-MRI is performed using a nonspecific extracellular contrast agent at a rate of $2-3 \mathrm{ml} / \mathrm{s}$, followed by approximately $40 \mathrm{ml}$ of $0.9 \%$ saline at the same rate via a catheter (20-22 G) placed in the cubital vein. The recommended total dose of the contrast agent is 0.025 or $0.05 \mathrm{mmol} / \mathrm{kg}$ body weight. Although good detectability of pancreatic cancer on gadoxetic acid-enhanced MRI was reported [12], it is difficult to assess delayed enhancement on these images. Similar to CT, DCE-MRI is obtained in multiple phases including the pancreatic phase (35-45 s after injection start) and delayed phase (130 s after injection start) [8]. A bolus tracking method is also recommended to obtain

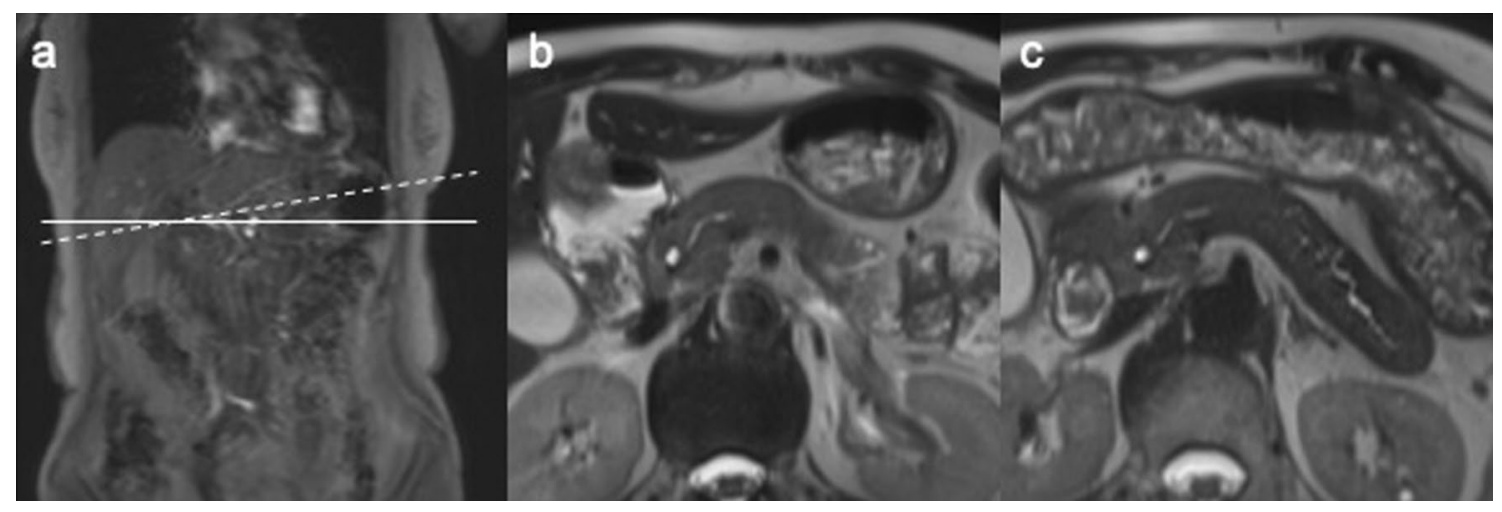

Fig. 1 Cross-sectional differences between axial and axial-oblique images obtained by ultrafast T2-weighted spin echo imaging (UFT2WI). a Two cross sections are shown on a reference image (white axial line and white oblique dotted line). b Axial UF-T2WI image. c Axial-oblique UF-T2WI image. The whole pancreas is shown 


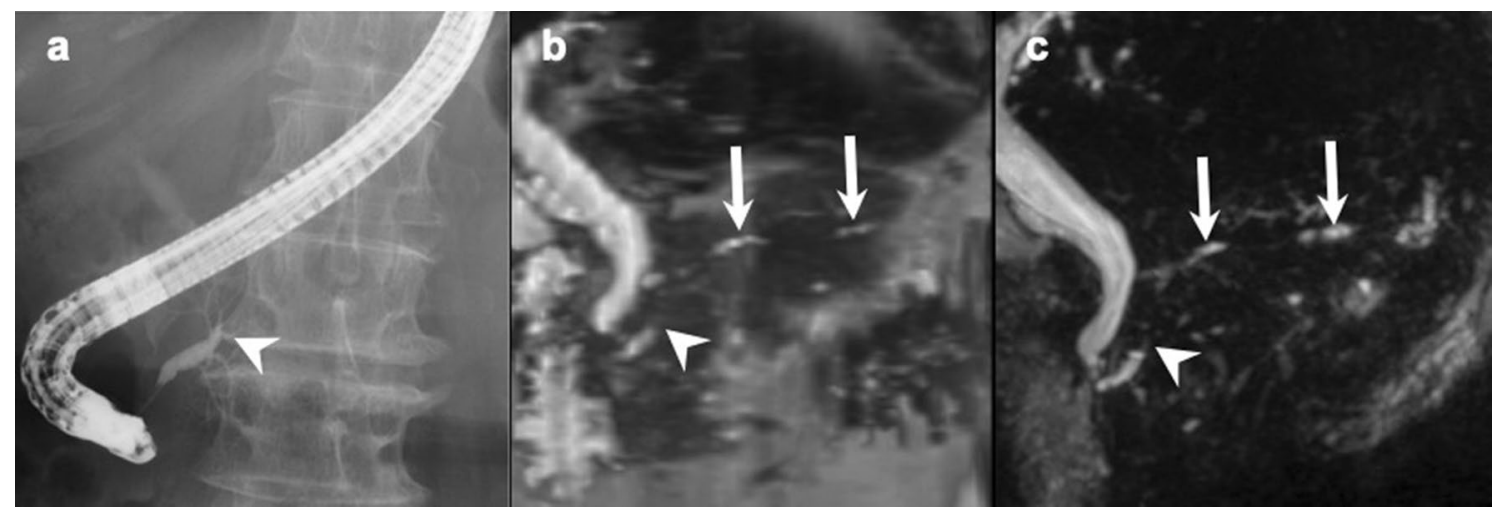

Fig. 3 Difference in depiction of the pancreatic duct (MPD) upstream of stenosis between magnetic resonance cholangiopancreatography (MRCP) and endoscopic retrograde pancreatography (ERP). a On an ERP image, the MPD downstream of the obstruction is vis- ualized (arrowhead). b, c The MPD in the pancreatic body and tail upstream of the obstruction (arrows) as well as the MPD downstream of the obstruction (arrowhead) are visualized on coronal ultrafast T2-weighted spin-echo imaging (b) and MRCP (c) images
Fig. 4 Usefulness of the partial maximum intensity projection (MIP) image on magnetic resonance cholangiopancreatography (MRCP). a An endoscopic retrograde pancreatography image shows branch ducts with narrowing (arrow) in a lesion of the pancreatic uncinate process. b Branch ducts are not seen on a coronal ultrafast $\mathrm{T} 2$-weighted spin-echo image (arrow). c Branch ducts are partially seen on a partial MIP image (arrow). d Branch ducts are clearly seen on a partial MIP image with a thickness of $10 \mathrm{~mm}$ (arrow)

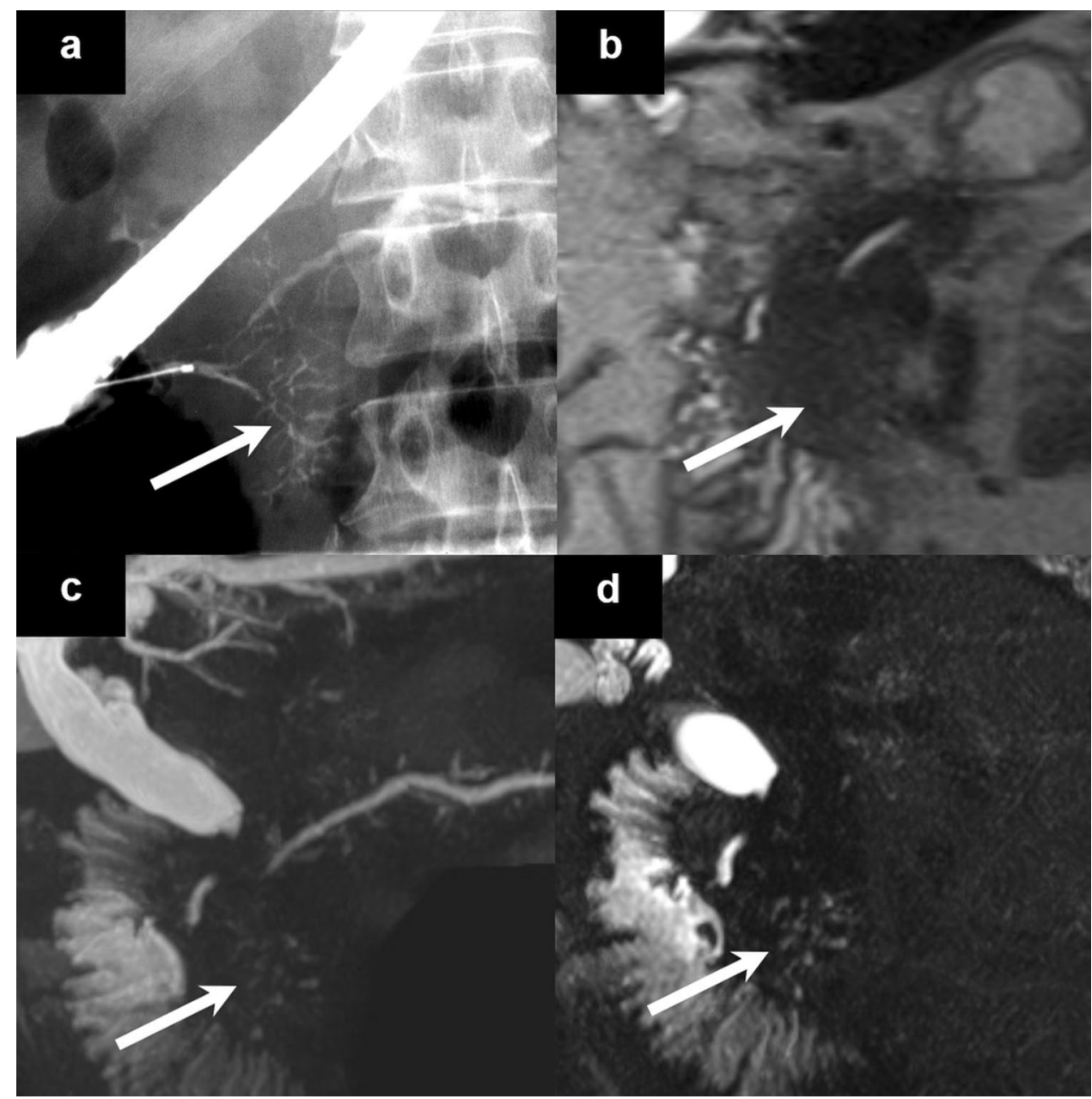

the optimal scan timing. High spatial and temporal resolution images can be obtained by time-resolved imaging or compressed sensing, and have the potential to improve the lesion detectability (Fig. 5).

\subsection{Important points for nuclear medicine examination}

On FDG-PET and Ga-67 scintigram, abnormal uptake is seen in a pancreatic lesion as well as in extrapancreatic 
Fig. 5 Advantage of high temporal resolution images for demonstrating speckled enhancement inside a lesion. a A pancreatic-phase dynamic contrast-enhanced image (24-s temporal resolution) shows pancreatic tail swelling and a slightly homogeneous hypovascular lesion. b On eight sub-frame images ( $3 \mathrm{~s}$ temporal resolution) of the pancreaticphase image, speckled/dotted enhancement is clearly seen on the first and second sub-frame images
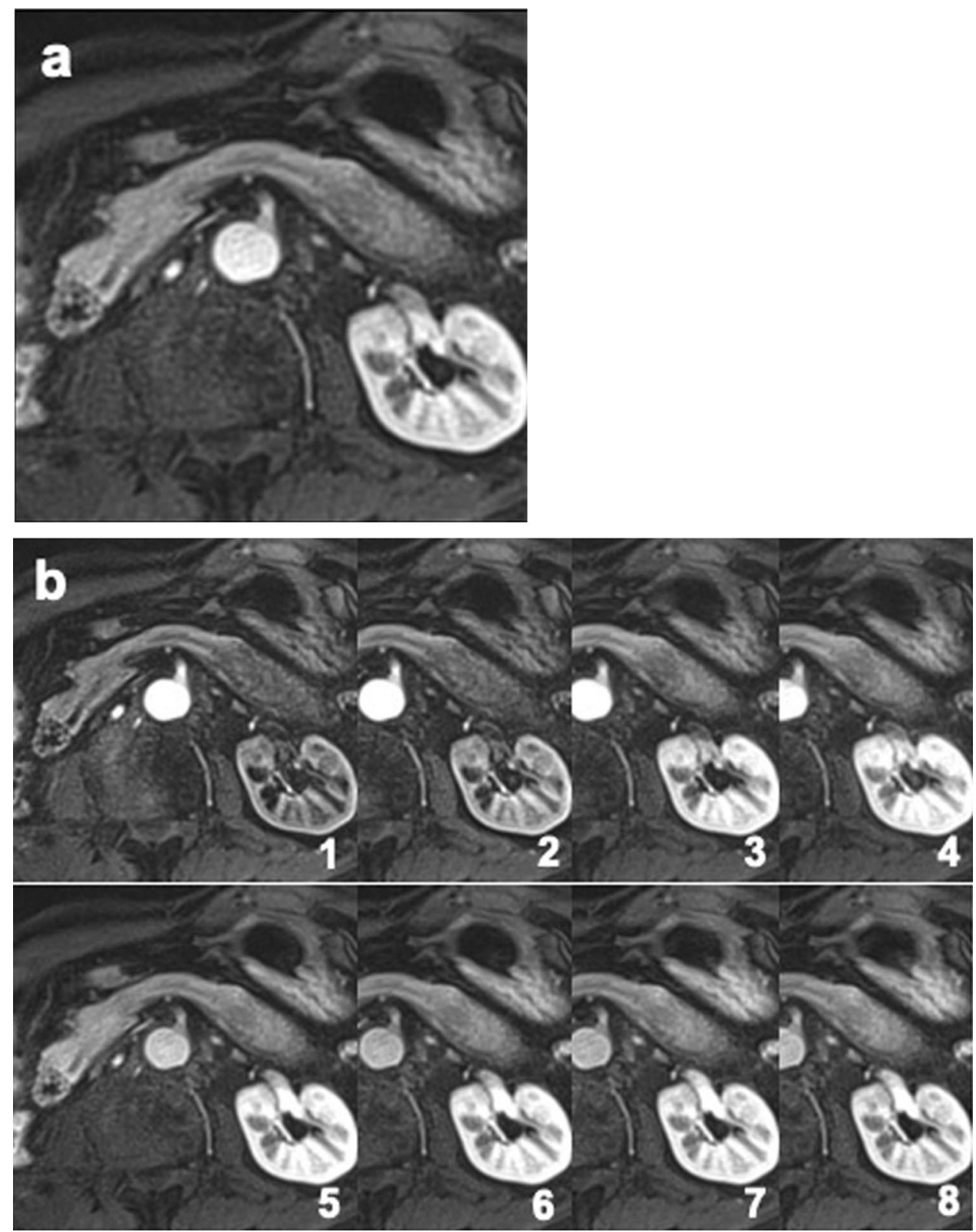

lesions. The scan range should be set with consideration of extrapancreatic lesions.

FDG-PET/CT and Ga-67 SPECT/CT are useful for accurate assessment of the accumulation site.

Standardized scan settings are better for comparisons between two examinations (e.g., before and after steroid therapy).

\subsubsection{Description}

Because IgG4-related disease is seen in organs throughout the body, whole-body (vertex to pelvic floor, at least) scans by FDG-PET and Ga-67 scintigraphy are recommended. A delayed FDG-PET scan is not mandatory, but is useful to discriminate between abnormal and physiological accumulation (e.g., accumulation in the bowel). In IgG4-related disease, the degree of FDG accumulation varies and there are no reference values for standardized uptake value $\left(\mathrm{SUV}_{\max }\right.$ and $\mathrm{SUV}_{\text {mean }}$ ) and metabolic tumor volume (MTV). However, FDG-PET can evaluate the metabolism in lesions, which is difficult to evaluate using other modalities (Fig. 6). FDG-PET is also useful for evaluating treatment effects (Fig. 7) and detecting exacerbations. However, it should be noted that FDG-PET for AIP is out of insurance coverage in Japan. 


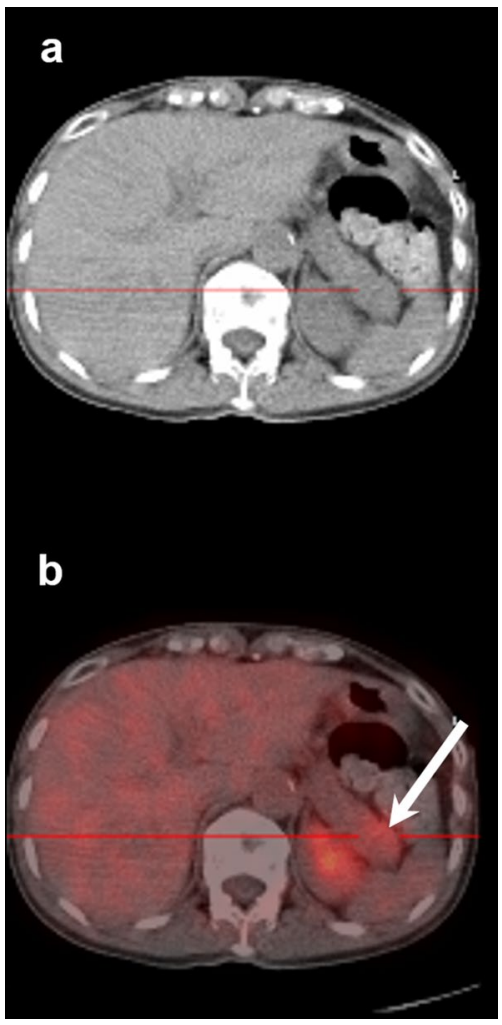

Fig. 6 Fluorodeoxyglucose-positron-emission tomography (FDGPET) findings of autoimmune pancreatitis (AIP). a The lesion is unclear on a non-contrast computed tomography (CT) image. b An FDG-PET/CT image shows nodular uptake (arrow) consistent with an AIP lesion in the pancreatic tail

\section{Radiologic findings for AIP}

\subsection{Summary of non-contrast CT and MRI findings (except for MRCP) (Figs. 8, 9, 10, 11)}

An AIP lesion is seen as a low-dense area on non-contrast $C T$, a hypointense area on FS-TIWI, and a hyperintense area on T2WI and DWI.

Speckled/dotted hyperintensity is sometimes seen in an AIP lesion on FS-TIWI.

The apparent diffusion coefficient (ADC) value is low.

\subsubsection{Description}

On non-contrast CT, an AIP lesion is seen as a low-dense area affected by infiltration of inflammatory cells such as lymphocytes and plasma cells (Fig. 8). The signal intensity of the normal pancreas is higher than that of the liver parenchyma on FS-T1WI and a signal decrease is observed with inflammation or loss of the normal pancreatic parenchyma (Fig. 9). Pathologically, a patchy distribution of the uninvolved pancreatic parenchyma in AIP was reported [13]. The speckled/dotted hyperintensity on FS-T1WI is thought to reflect the pathologic findings (Fig. 9) [14, 15]. On FST2WI, AIP is usually shown as a hyperintense area, but the signal decreases as the fibrosis increases. UF-T2WI is sometimes more useful than MRCP because the association between the lesion and the pancreatic duct is clearly shown. On DWI, an AIP lesion usually appears as a hyperintense
Fig. 7 Decrease in Ga-67 accumulation after steroid treatment. a Before treatment, the head of the pancreas (arrow) is swollen and abnormal uptake is seen. $\mathbf{b}$ After steroid treatment, both the enlargement and accumulation are improved (arrowhead)

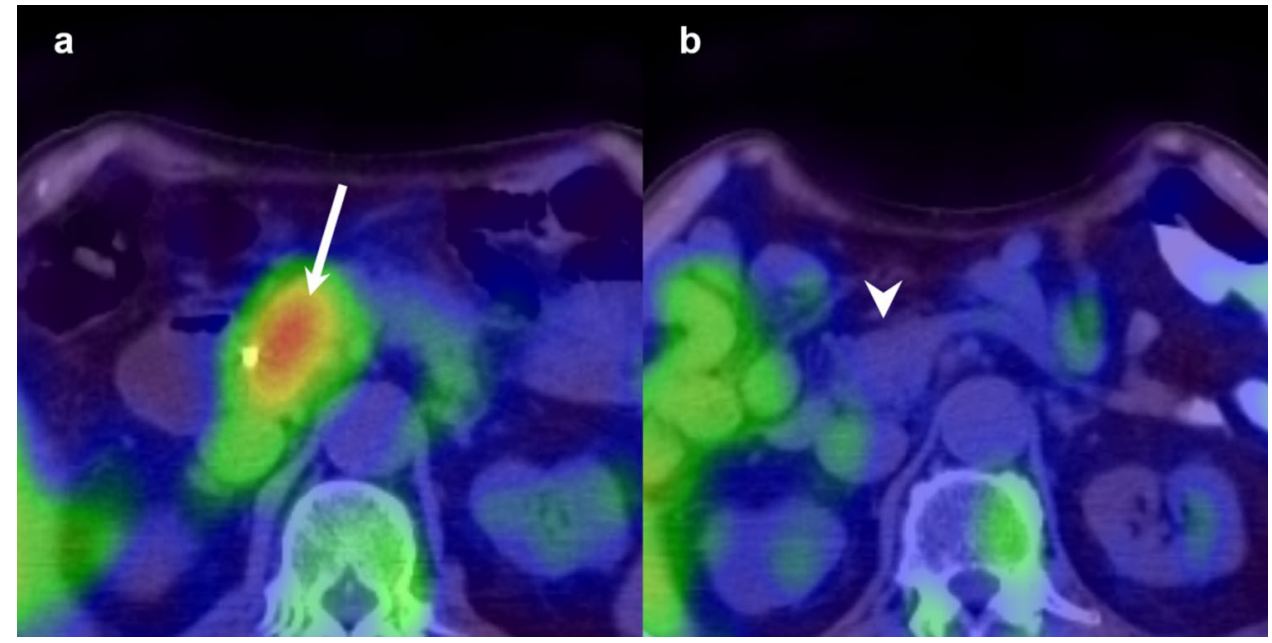




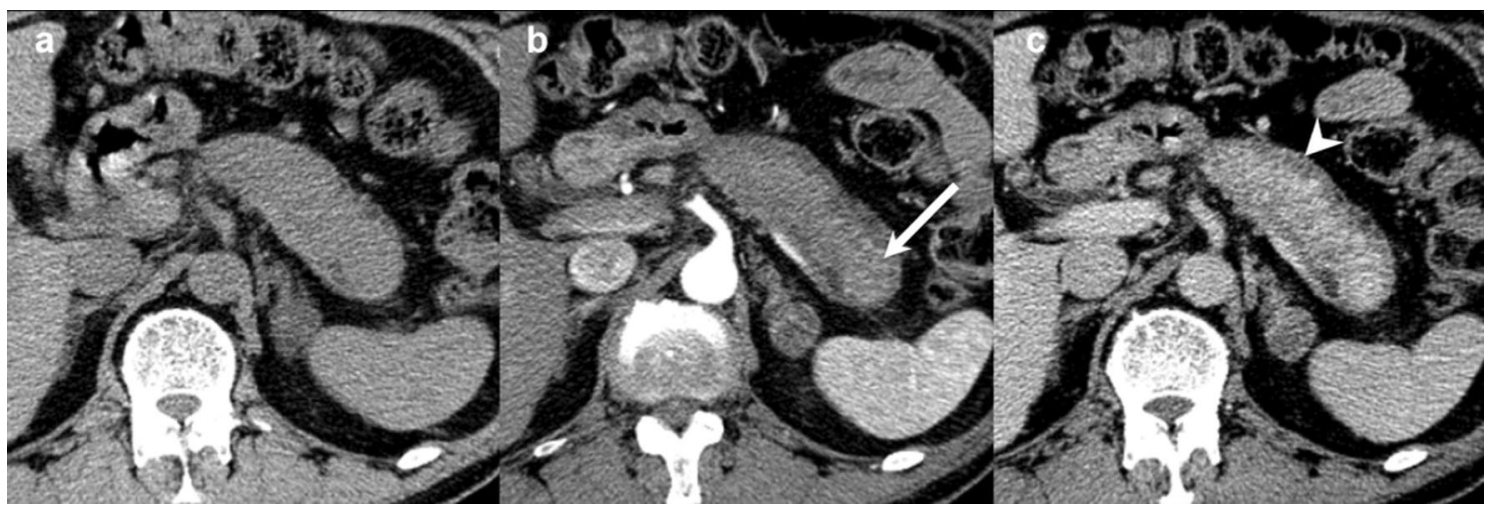

Fig. 8 Typical computed tomography (CT) findings of autoimmune pancreatitis (AIP). a A non-contrast CT image shows diffuse pancreatic enlargement with decreasing density, smooth margin (sausage-like appearance), and loss of the cobblestone structure in the pancreas. b A pancreatic-phase dynamic contrast-enhanced
CT (DCE-CT) image shows that a hypovascular lesion with patchy enhancement (arrow). c A delayed-phase DCE-CT image shows delayed enhancement of the AIP lesion and a capsule-like rim (arrowhead). The main pancreatic duct is unclear, but there is no dilation

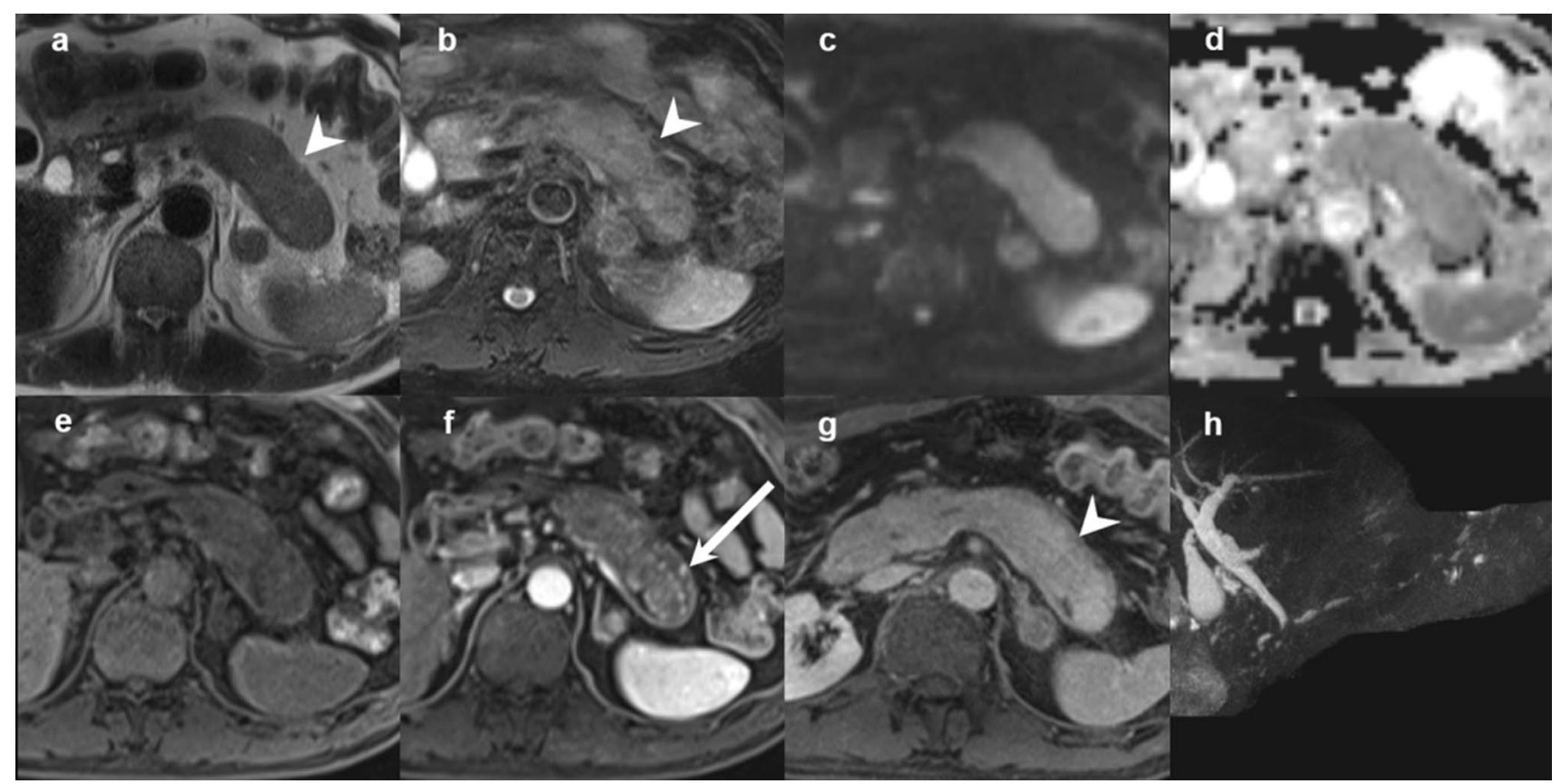

Fig. 9 Typical magnetic resonance (MR) findings of autoimmune pancreatitis (AIP) (same case as Fig. 8). a An ultrafast T2-weighted spin-echo image shows sausage-like swelling (diffuse swelling and loss of the cobblestone structure in the pancreas) and a capsule-like rim (band-like hypointense area around the pancreas; arrowhead) in the tail of the pancreas. b The capsule-like rim is slightly unclear on a fat-suppressed T2-weighted spin-echo image. c A diffusion-weighted image $(b=1000)$ shows the AIP lesion as a diffuse hyperintense area. d An apparent diffusion coefficient map shows restricted diffusion in the lesion equivalent to the spleen. e A fat-suppressed T1-weighted imaging (FS-T1WI) image shows a diffuse hypointense lesion, but dotted hyperintense areas within the lesion (dotted hyperintensity on FS-T1WI). f A pancreatic-phase dynamic contrast-enhanced MR (DCE-MR) image reveals that the areas shown as dotted hyperintensity on FS-T1WI are enhanced (speckled/dotted enhancement; arrow). g A delayed-phase DCE-MR image shows homogeneous delayed enhancement of the AIP lesion and a capsule-like rim (arrowhead). $\mathbf{h}$ A magnetic resonance cholangiopancreatography image shows multiple skipped narrowing (skip lesions) of the main pancreatic duct (MPD) without dilation of the upstream MPD (no MPD dilatation) 


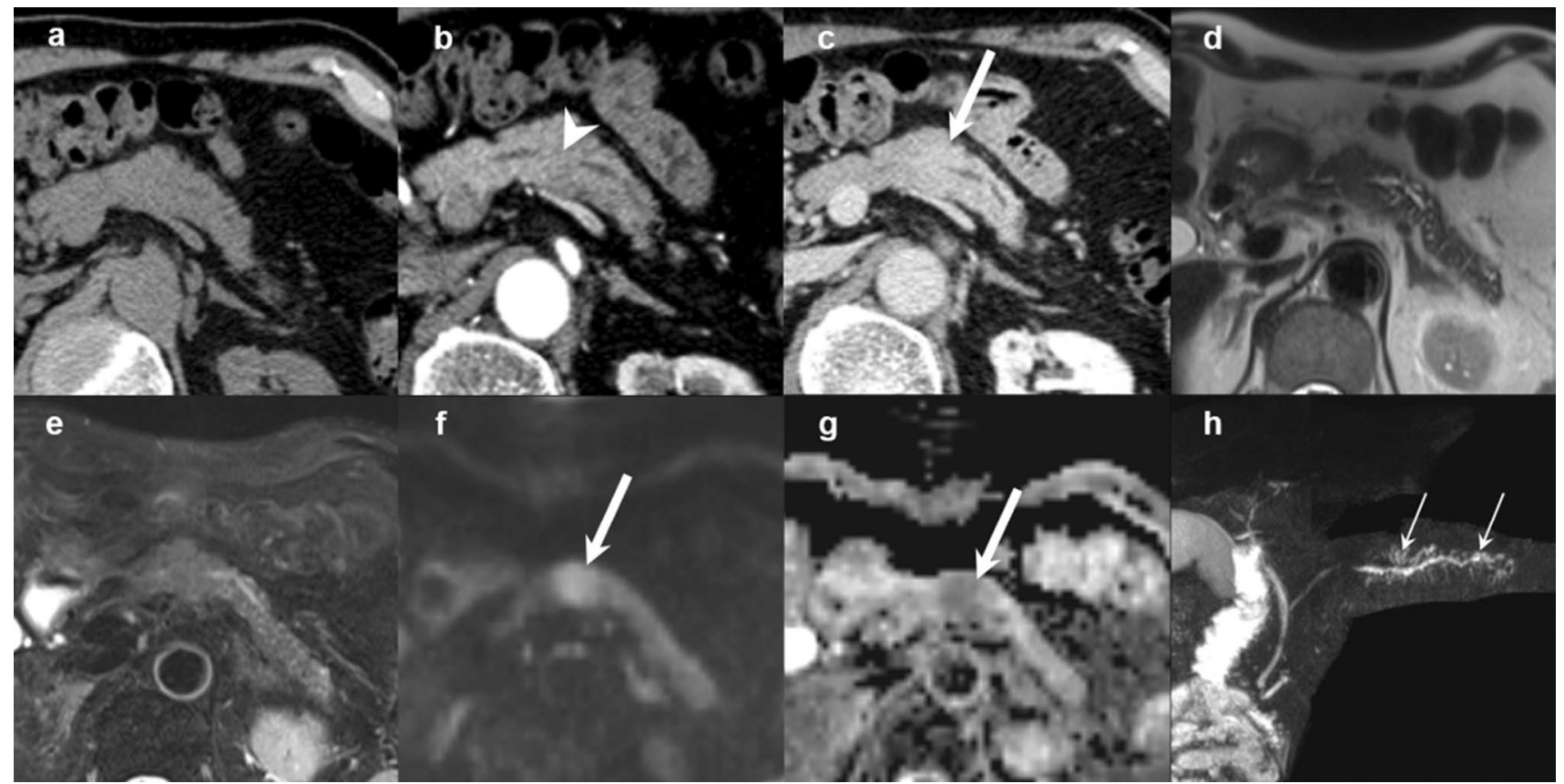

Fig. 10 Computed tomography (CT) and magnetic resonance (MR) images of focal autoimmune pancreatitis. a The lesion is unclear on a non-contrast CT image. b On a pancreatic-phase of dynamic contrast-enhanced CT (DCE-CT) image, the main pancreatic duct (MPD) is partially unclear (arrowhead) in the body of the pancreas, while the mass lesion is unclear. c On a delayed-phase DCE-CT image, a faint nodule-like enhancement is observed in the pancreatic body (arrow). d, e On an ultrafast T2-weighted spin-echo image (d) and a fat-suppressed T2-weighted spin-echo image (e), the MPD is partially unclear in the pancreatic body, but the mass lesion is unclear. $\mathbf{f}$ A diffusion-weighted image $(b=1000)$ shows a nodular hyperintense area (arrow) in the pancreatic body. $\mathbf{g}$ An apparent diffusion coefficient map shows restricted diffusion with a decreased signal intensity area (arrow). h A magnetic resonance cholangiopancreatography image shows stenosis at the same site, and the branch ducts are slightly dilated (small arrows)

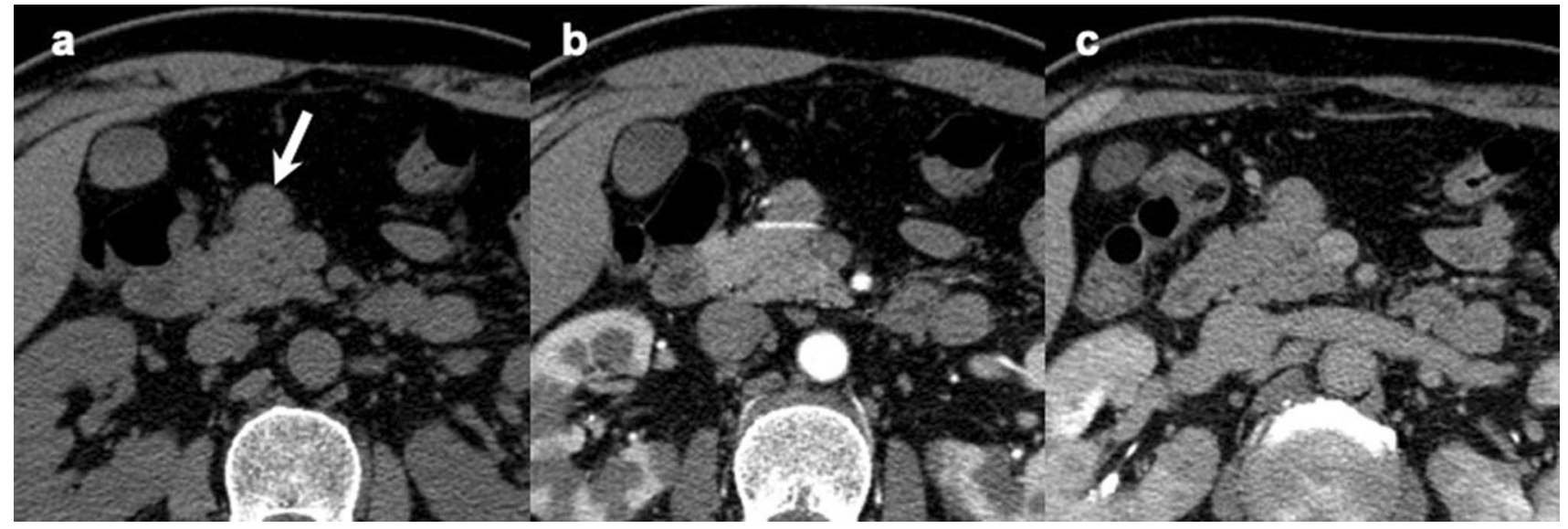

Fig. 11 Computed tomography (CT) imaging of nodule-like autoimmune pancreatitis. a A non-contrast CT image shows a nodule-like lesion (arrow) protruding ventrally from the pancreatic head. b On a pancreatic-phase dynamic contrast-enhanced CT (DCE-CT) image, the lesion is slightly enhanced. c On a delayed-phase DCE-CT image, the lesion is homogeneously enhanced area (Fig. 9). The ADC value of AIP is lower than that of pancreatic cancer [16-19]. However, cutoff values are not useful for differentiation because they vary among reports based on different MR units and scan parameters.

\subsection{Pancreatic swelling (diffuse and focal) [1]}

Pancreatic enlargement is assessed by the Haaga criteria (enlargement of more than one vertebra in the pancreatic head or enlargement of more than 2/3 vertebra in the 
pancreatic body and tail are recognized as pancreatic swelling) [20].

Although there are no strict definitions of "diffuse" and "focal", the following definitions are commonly used: focal, $<1 / 3$, segmental, $>1 / 3$ to $<2 / 3$, diffuse $>2 / 3$.

Multiple focal lesions may appear simultaneously.

\subsubsection{Description}

Diffuse or focal pancreatic swelling is caused by marked lymphocyte and plasma cell infiltration and fibrosis. In the elderly, the margin of the pancreas is lobulated and the inside structure of the pancreas has a cobblestone appearance. However, the margin of the pancreas is straightened (termed "sausage-like appearance") in AIP patients (Figs. 8, 9). It is difficult to define the normal size of the pancreas because it is affected by age and fatty infiltration. If pancreatic swelling is unclear at the onset of AIP, it may become obvious with consideration of the morphologic changes of the pancreas after steroid therapy. Furthermore, the extent of the pancreatic swelling depends on the degree of inflammation. Therefore, pancreatic swelling should be assessed in consideration of the focal changes in density/signal and contrast effect if the pancreatic swelling is unclear (Figs. 10, $11,12)$.

\subsection{Speckled/dotted hyperintensity on FS-T1WI and speckled/dotted enhancement on the early phase of DCE-MRI}

In an AIP lesion, speckled/dotted hyperintensity is seen on FS-TIWI and the area is enhanced (speckled/dotted enhancement) on the pancreatic phase of DCE-MRI [14, $15]$.

\subsubsection{Description}

As described in Section 2.1, patchy distribution of the uninvolved pancreatic parenchyma is seen and appears as a speckled/dotted hyperintense area on FS-T1WI (Figs. 9, 13). The area is well enhanced on the pancreatic phase of DCEMRI because the normal pancreas has abundant blood flow (Figs. 9, 13). A fat-suppression technique is useful because it improves the contrast between the lesion and the adjacent pancreatic parenchyma and allows observation of the detailed changes in the pancreas.

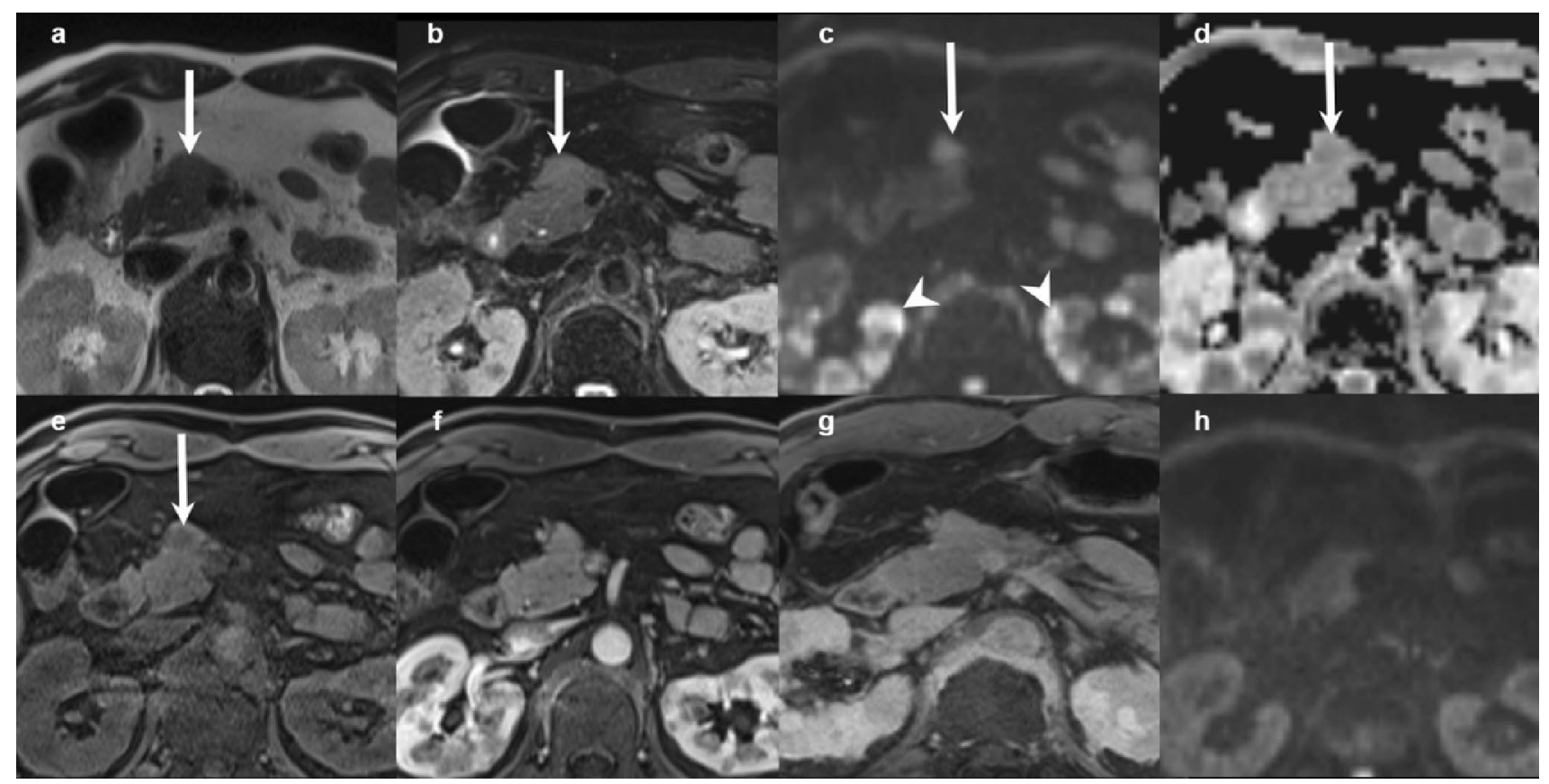

Fig. 12 Magnetic resonance (MR) images of nodule-like autoimmune pancreatitis (same case as Fig. 11). a-e A nodule-like lesion (arrow) protruding ventrally from the head of the pancreas is seen as a slightly hyperintense area on an ultrafast T2-weighted spin-echo image (a), fat-suppressed T2-weighted spin echo image (b), and diffusion-weighted image (DWI) $(b=1000)(\mathbf{c})$, and as a slightly hypointense area on an apparent diffusion coefficient map (d) and fat-suppressed T1-weighted image (e). f, $\mathbf{g}$ The lesion is enhanced as well as the pancreatic head on a pancreatic-phase dynamic contrast-enhanced MR (DCE-MR) image (f) and shows homogeneous delayed enhancement on a delayed-phase DCE-MR image (g). h A DWI image after steroid treatment shows disappearance of the lesion. In the bilateral kidneys, multiple nodule-like lesions associated with IgG4-related disease are observed. These lesions are seen as hyperintense areas (arrowhead) on the DWI (c), and were improved after steroid treatment (h) 


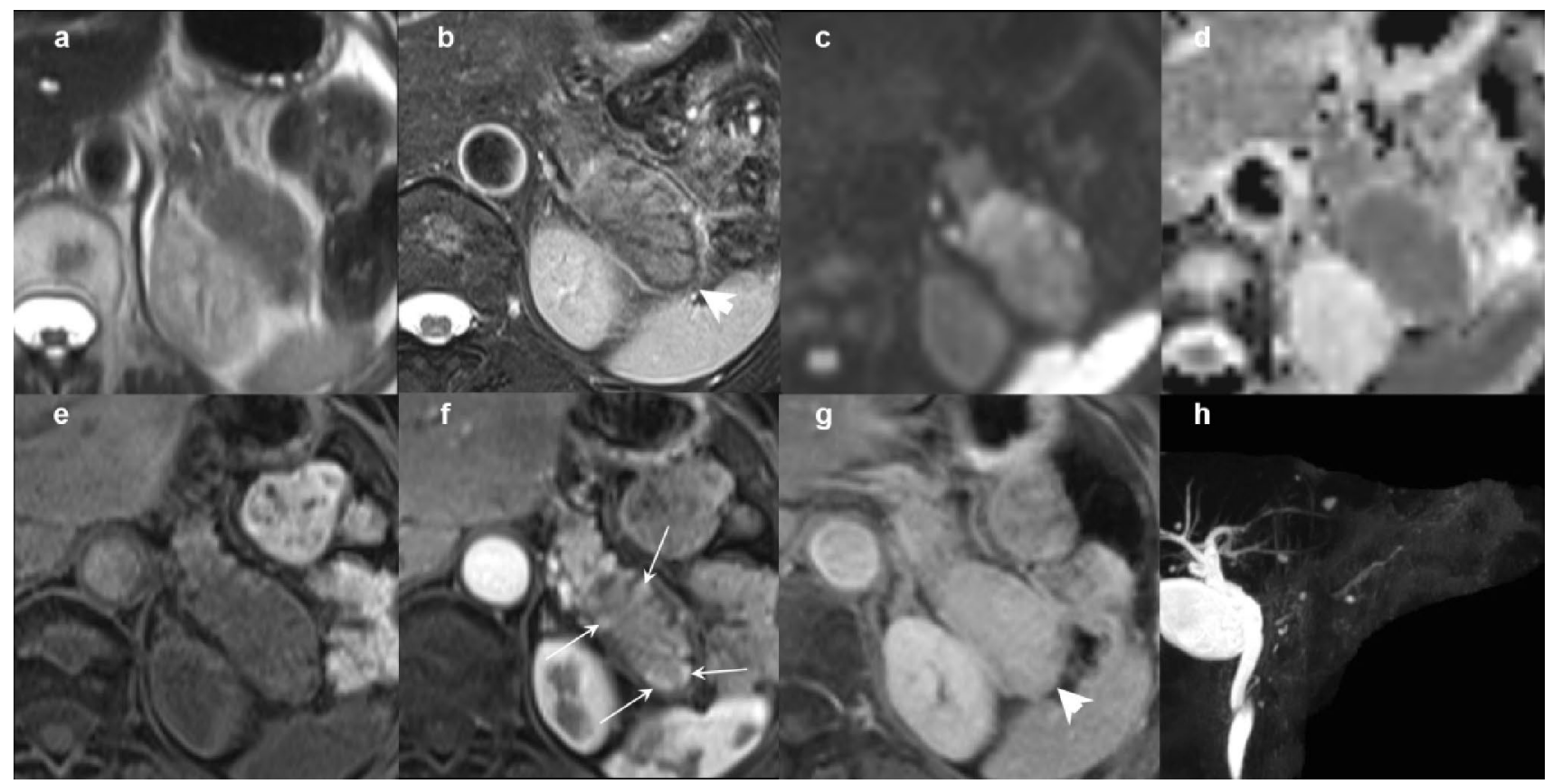

Fig. 13 Typical magnetic resonance (MR) findings for focal autoimmune pancreatitis (AIP). a, b Pancreatic tail enlargement is seen on an ultrafast T2-weighted spin-echo image (a) and fat-suppressed T2-weighted spin-echo image (FS-T2WI) (b). A capsule-like rim (band-like hypointense area around the pancreas; arrowhead) is also seen on the FS-T2WI (arrowhead). c A diffusion-weighted image $(b=1000)$ shows a nodular hyperintense area corresponding to the AIP lesion. d an apparent diffusion coefficient map shows restricted diffusion in the lesion, appearing as a hypointense area. e, $\mathbf{f}$ A fat-suppressed T1-weighted image shows decreased signal intensity in the
AIP lesion with speckled/dotted hyperintense areas within the lesion (e) and these areas are enhanced (speckled/dotted enhancement; small arrows) on a pancreatic-phase dynamic contrast-enhanced MR image (f). g A delayed-phase of dynamic contrast-enhanced computed tomography image shows homogeneous delayed enhancement of the AIP lesion as well as a capsule-like rim (arrowhead). h The main pancreatic duct (MPD) shows irregular narrowing with no dilation of the upstream MPD (no MPD dilatation) on a magnetic resonance cholangiopancreatography image

on DCE-CT/MRI (Figs. 8, 9, 13, 14) [8, 23, 24]. The capsule-like rim was reported to reflect dense fibrosis around the lesion, but its frequency varied among different reports [23-25]. The finding is never seen in diseases other than AIP and is one of the specific findings that can differentiate AIP from pancreatic cancer [16, 17, 21, 26-28]. However, its sensitivity is low when a lesion is small [15].

\subsection{Homogeneous delayed enhancement}

AIP is hypovascular on the pancreatic phase of DCE-CT/ $M R I$ and homogeneously enhanced on the delayed phase of DCE-CT/MRI.

\subsubsection{Description}

Homogeneous delayed enhancement is affected by loss of normal pancreatic acini and extensive fibrosis. Delayed enhancement is seen in pancreatic cancer. However, the enhancement is commonly inhomogeneous due to necrosis, hemorrhage, and degeneration in the tumor. Therefore, homogeneous delayed enhancement is one of the specific 


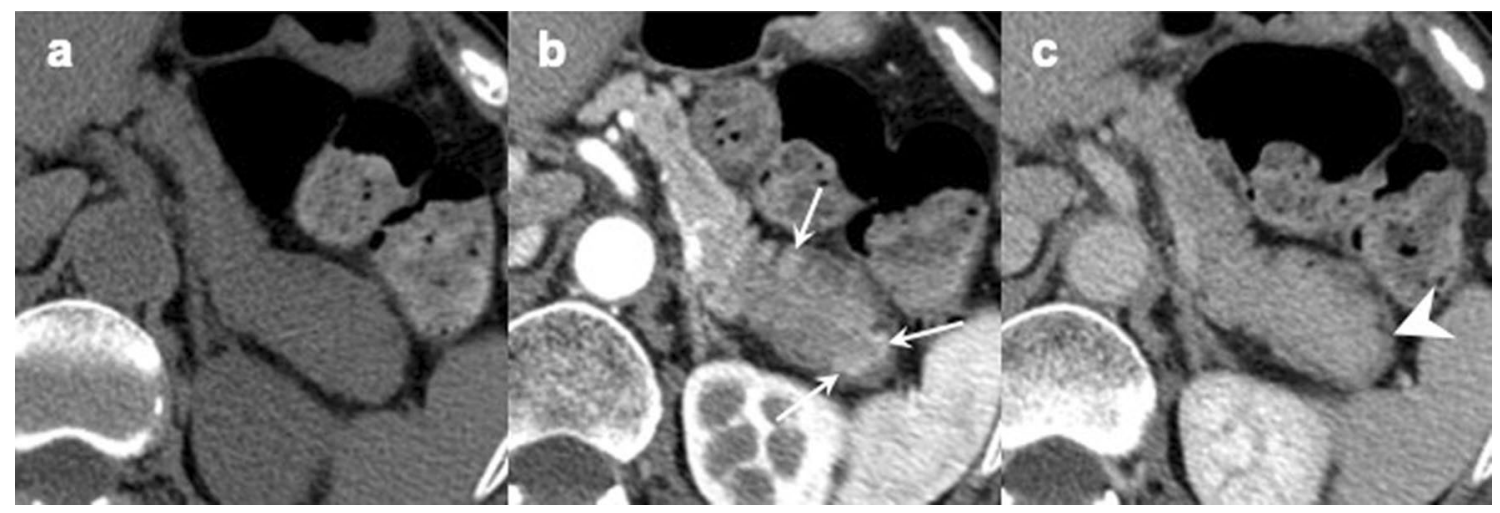

Fig. 14 Typical computed tomography (CT) findings of focal autoimmune pancreatitis (AIP) (same case as Fig. 11). a The pancreatic tail is enlarged like a mass and presents low density. The cobblestone appearance is unclear in this area. b A pancreatic-phase dynamic contrast-enhanced CT (DCE-CT) image shows mild con- trast enhancement as a whole, but a point-like or nodular enhancement area remains (speckled/dotted enhancement; arrow) inside the AIP lesion. c A delayed-phase DCE-CT image shows homogeneous delayed enhancement of the AIP lesion and delayed enhancement of the capsule-like rim (arrowheads)
Fig. 15 Duct-penetrating sign. a A fat-suppressed T1-weighted image shows segmental pancreatic tail swelling and speckled/ dotted hyperintense areas within the lesion (small arrows). b On an ultrafast T2-weighted spinecho image, the main pancreatic duct is visible and running through the lesion without obstruction (arrow)
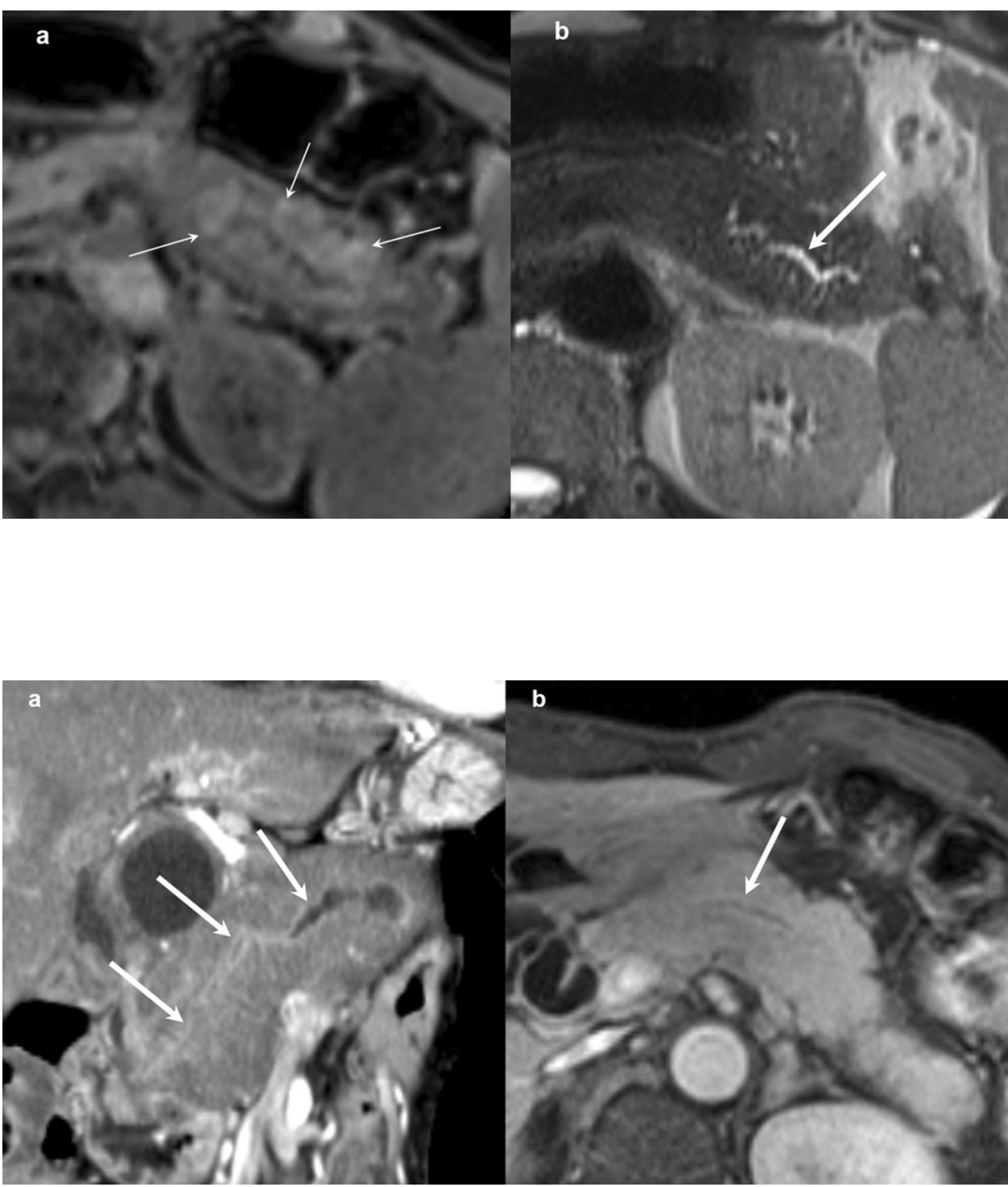

Fig. 16 Enhanced duct sign. a A curved MPR image during the pancreatic phase of dynamic contrast-enhanced computed tomography shows diffuse pancreatic swelling and the enhanced duct sign along the main pancreatic duct (arrows). b The enhanced duct sign is also clearly seen on a delayed-phase contrast-enhanced magnetic resonance image (arrow) 


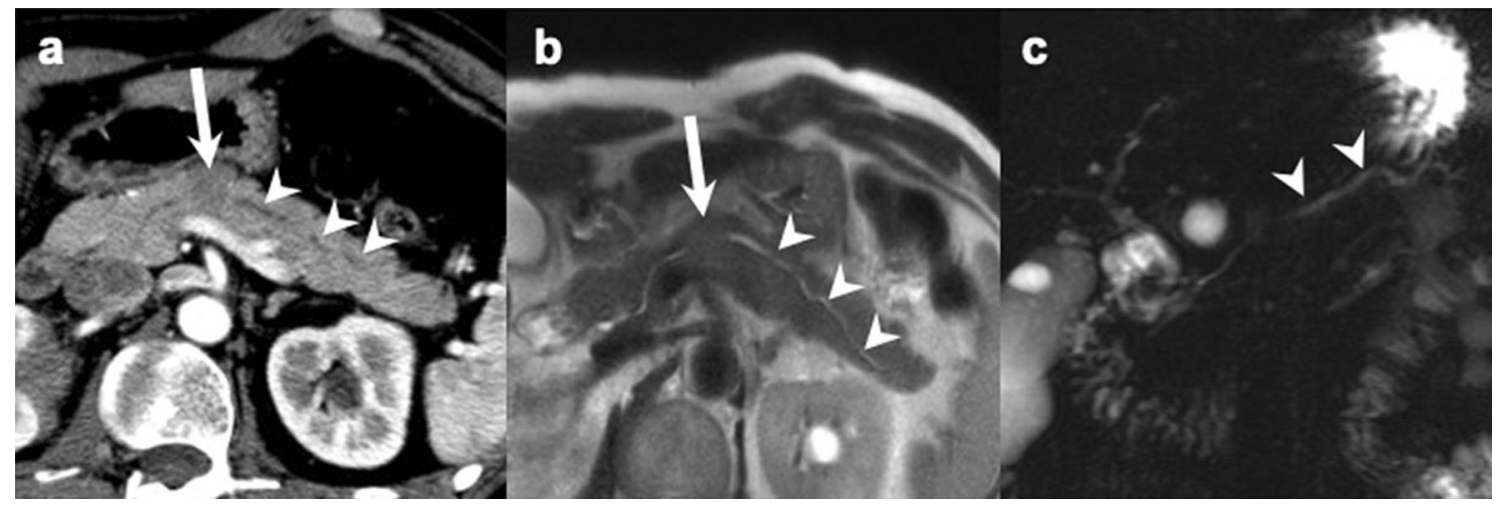

Fig. 17 No main pancreatic duct (MPD) dilatation. a, b A focal autoimmune pancreatitis (AIP) lesion (arrow) is observed in the pancreatic body as a hypovascular nodule on a pancreatic-phase dynamic contrast-enhanced computed tomography image (a) and a slightly

findings of AIP, and is useful for differentiation between AIP and pancreatic cancer (Figs. 8, 9, 10, 11, 12, 13, 14) [15, 16, $18,26,28,29]$. It should be noted that delayed enhancement is not seen in AIP when the amount of fibrosis is low.

\subsection{Duct-penetrating sign, icicle sign, and skipped narrowing}

The MPD sometimes penetrates an AIP lesion [30], and sometimes tapers like an icicle [27].

Pancreatic duct narrowing is a characteristic finding of AIP and is sometimes skipped [9, 10, 31].

\subsubsection{Description}

The duct-penetrating sign is one of the useful findings for differentiation of AIP from pancreatic cancer, but its sensitivity is low (Fig. 15) [16, 18, 21, 27, 30]. Pancreatic duct hyperintense area on an ultrafast T2-weighted spin-echo image (UFT2WI) (b). b, c The MPD (arrowheads) is unclear in the AIP lesion, but upstream MPD dilatation is not seen on the UF-T2WI (b) or a magnetic resonance cholangiopancreatography image (c)

narrowing is frequently seen in AIP, but the duct is rarely obstructed. However, no significant difference in the frequency of the duct-penetrating sign was found when the lesions were small [9]. Previously, assessment of the pancreatic duct on MRCP was not permissible because the image quality was unsatisfactory compared with ERP [9]. Although the image quality on MRCP currently remains inferior to that on ERP, it has been improved and MRCP was adopted in the JPS2018 [1]. The partial MIP is one of the useful methods for visualization of small pancreatic ducts including the branches (Fig. 4) [11]. Breath-hold compressedsensing accelerated 3D MRCP added to respiratory-gating conventional MRCP can sometimes compensate for poor image quality due to motion artifacts [32]. The pancreatic duct findings sometimes differ on ERP and MRCP because the contrast agent is injected into the pancreatic duct in ERP and motion artifacts can affect MRCP [11, 33]. MPD narrowing is sometimes seen as a disruption on ERP or a
Table 1 Extrapancreatic lesions associated with autoimmune pancreatitis

\begin{tabular}{ll}
\hline Close association & Possible association \\
\hline Lachrymal gland inflammation [54] & Hypophysitis [55] \\
Sialadenitis [54] & Pachymeningitis [56] \\
Hilar lymphadenopathy [57] & Autoimmune neurosensory hearing loss [42] \\
Interstitial lung disease [58, 59] & Uveitis [60] \\
Sclerosing cholangitis [61] & Chronic thyroiditis [62, 63] \\
Retroperitoneal fibrosis [64] & Pseudotumor (breast, lung, liver) [65-67] \\
Tubulointerstitial nephritis [68, 69] & Gastric ulcer [70] \\
Periaortitis/periarteritis [71, 72] & Swelling of papilla of Vater [73] \\
& IgG4 hepatopathy [74, 75] \\
& Prostatitis [76] \\
IgG4-related perineural disease [77, 78] \\
IgA vasculitis (Schönlein-Henoch purpura) [42] \\
Autoimmune thrombocytopenia [79]
\end{tabular}




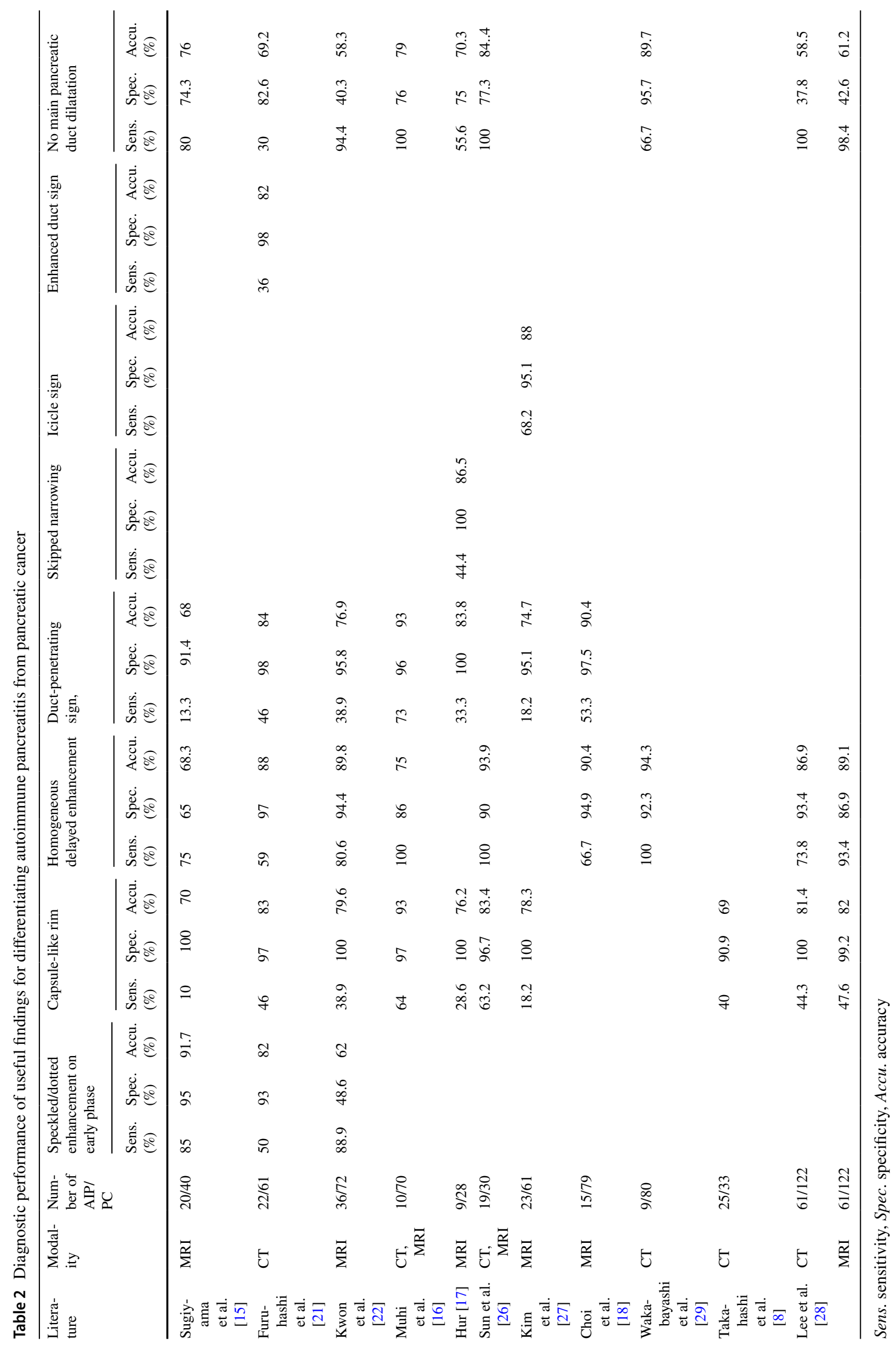


non-visualized area on MRCP. Skipped narrowing of the MPD is a useful finding for differentiation of AIP from pancreatic cancer because its sensitivity and specificity were reported to be $44.4-47.5 \%$ and $97.5-100 \%$, respectively [17, $28,31]$.

\subsection{Enhanced duct sign}

Linear enhancement along the MPD is sometimes seen in an AIP lesion.

\subsubsection{Description}

The enhanced duct sign was reported to be a useful finding for differentiation of AIP from pancreatic cancer (Fig. 16), with sensitivity and specificity of $67 \%$ and $95 \%$, respectively [34]. However, the sensitivity of the enhanced duct sign in small AIP lesions was not very high [21]. This finding may reflect inflammation around the MPD.

\subsection{No MPD dilatation}

In AIP, MPD dilatation is not seen or rarely seen in the upstream of the lesion.

\subsubsection{Description}

The definition of MPD dilation varies from 3 to $5 \mathrm{~mm}$ in diameter and there are no established criteria for this finding. On ERP, one of the characteristic findings for AIP is no

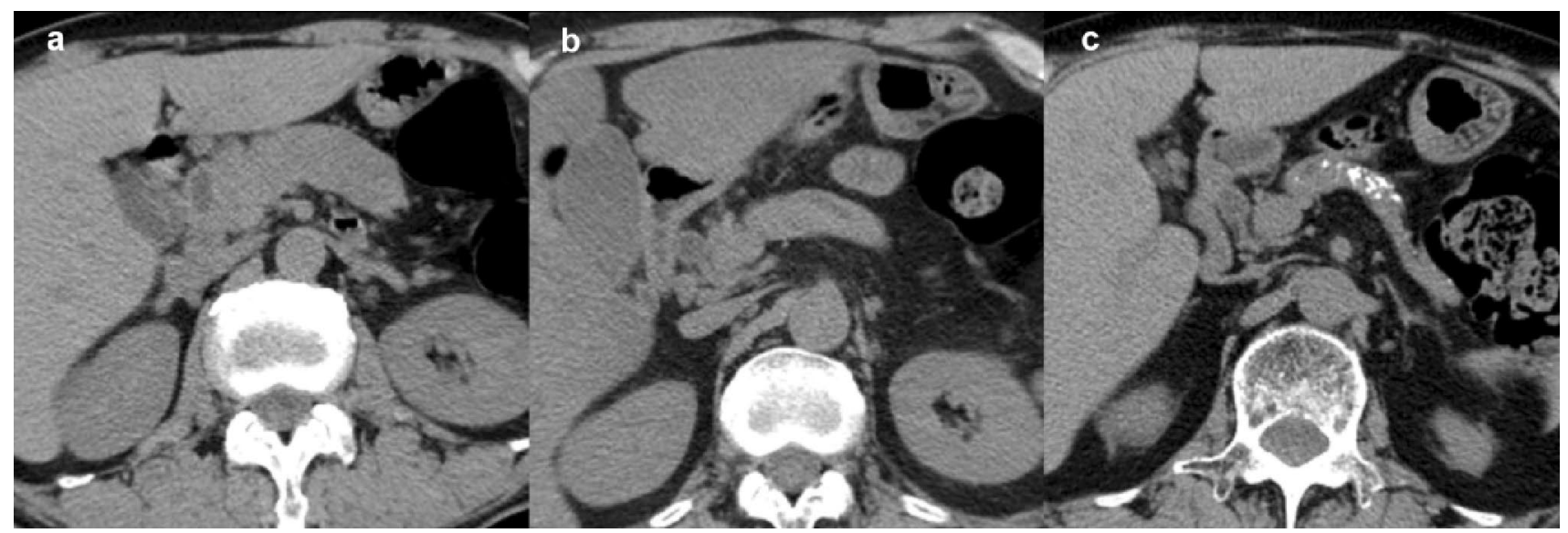

Fig. 18 Pancreatic atrophy and calcification in autoimmune pancreatitis (AIP) patients with repeated relapses. a A non-contrast computed tomography (CT) image shows diffuse pancreatic swelling. b A noncontrast CT image at 10 years after steroid treatment shows improve- ment of the pancreatic swelling. c A non-contrast CT image after multiple relapses (6 years and 7 months after the initial treatment) shows atrophy of the pancreas and multiple calcifications

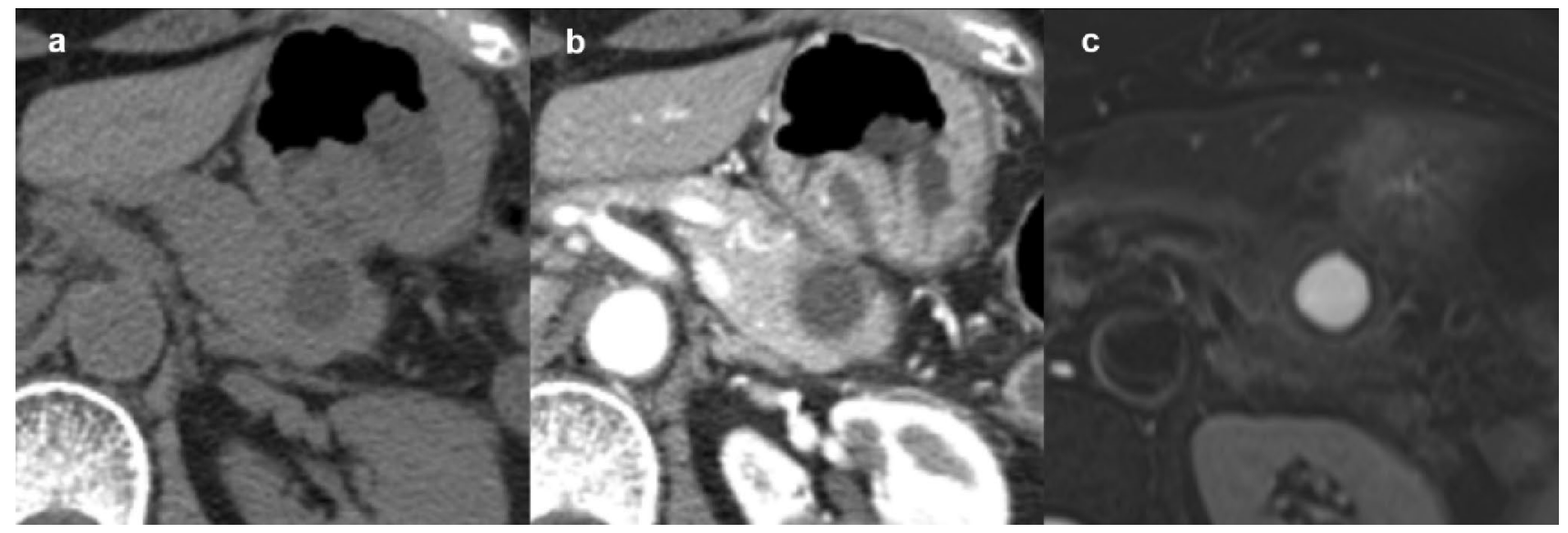

Fig. 19 Autoimmune pancreatitis (AIP) with cyst formation. a A noncontrast computed tomography (CT) image shows cyst formation in the enlarged pancreatic tail (arrow). b, c No enhancement of the cyst formation is seen on a pancreatic-phase dynamic contrast-enhanced CT image (b), and the lesion is seen as a markedly hyperintense area on a fat-suppressed T2-weighted spin-echo image (c) 
Fig. 20 Diffuse autoimmune pancreatitis (AIP) with splenic vein occlusion and encasement of the splenic artery on the pancreatic phase of dynamic contrast-enhanced computed tomography (DCE-CT). a A contrast-enhanced CT image shows a sausage-like appearance and a capsule-like rim of the pancreas. Irregularity and disruption (arrow) of the splenic vein are seen, and development of a collateral tract around the stomach (arrowhead) is also seen. b Encasement of the splenic artery (white open arrow) is seen at the upper level of (a). c After steroid treatment, a contrast-enhanced CT image shows improvement of the splenic vein occlusion (arrow) and collateral circulation (arrowhead). d The encasement of the splenic artery (white open arrow) is also improved at the upper level of (c)

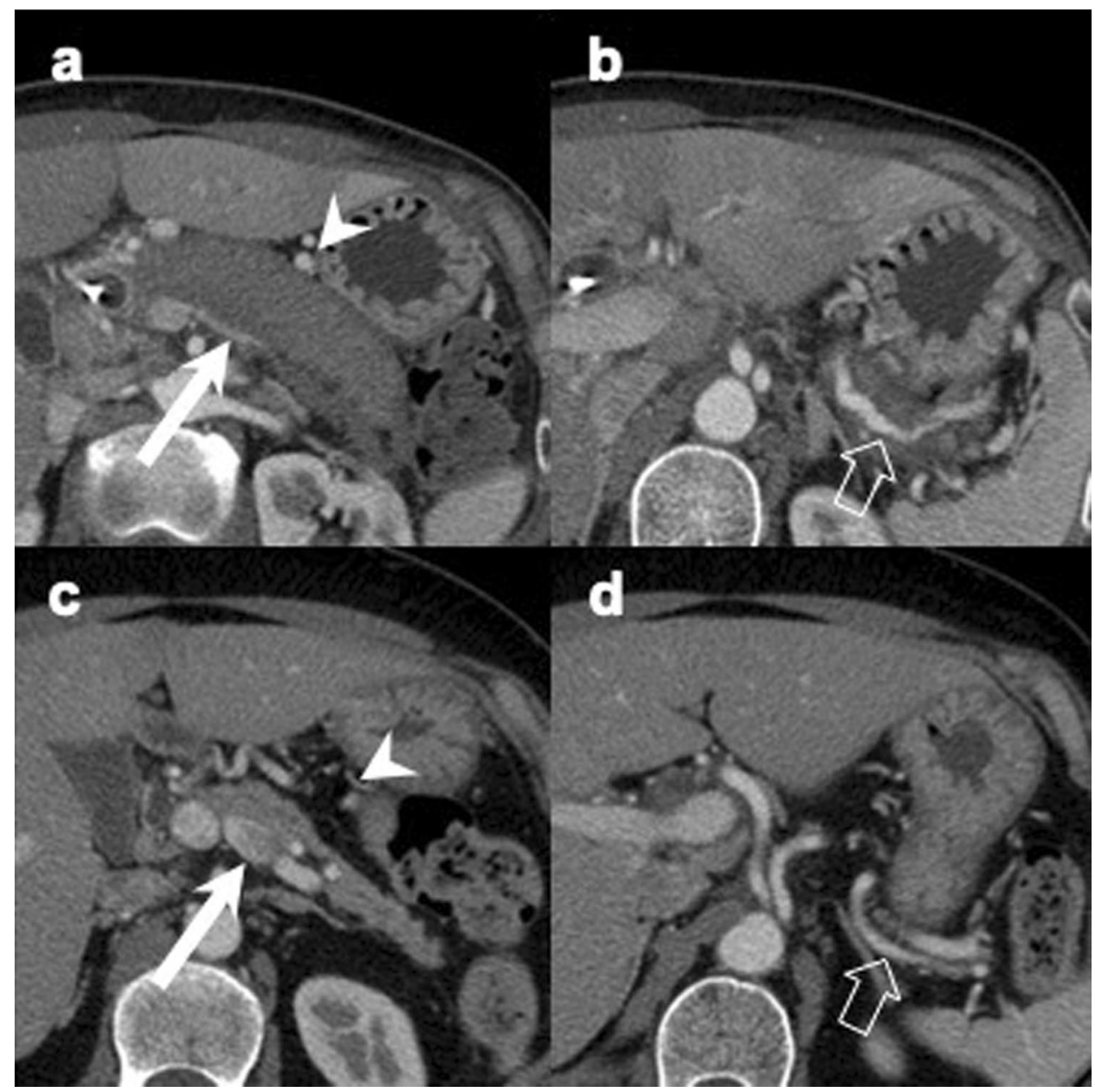

MPD dilatation ( $\leq 4 \mathrm{~mm}$ in diameter) in the upstream of the lesion (Fig. 17) [28, 29, 35]. It should be noted that MPD dilatation may be seen if there is atrophy of the pancreatic parenchyma.

\subsection{Findings for nuclear medicine examination (FDG-PET and Ga-67 scintigram)}

The degrees of drug accumulation in AIP and extrapancreatic lesions depend on the lesion activity.

The drug accumulation decreases if treatment is effective.

\subsubsection{Description}

FDG-PET and Ga-67 scintigram are useful for diagnosis of AIP because they can evaluate the lesion function [36, 37], which cannot be evaluated by CT and MRI. In AIP patients, inhomogeneous or multifocal FDG accumulation is seen on FDG-PET, and uptake in extrapancreatic lesions is also seen [38-40]. The degrees of drug accumulation in AIP and extrapancreatic lesions depend on the lesion activity, and it is necessary to make a careful diagnosis using multiple modalities for differentiation between physiological and pathological uptake. The usefulness of FDG-PET/CT and Ga-67 SPECT/CT as well as the usefulness of nuclear medicine examinations for response evaluation are described in Section 1.4 (Fig. 7).

\subsection{Extrapancreatic lesions}

IgG4-related sclerosing cholangitis, sclerosing dacryoadenitis/sialadenitis, retroperitoneal fibrosis, and kidney disease are described in the JPS2018.

\subsubsection{Description}

IgG4-related kidney disease, which was already described in the International Consensus Diagnostic Criteria (ICDC) for AIP [41], was added to IgG4-related sclerosing cholangitis, sclerosing dacryoadenitis/sialadenitis, and retroperitoneal fibrosis in the JPS2018. Many other extrapancreatic lesions can be found throughout the body $[6,42,43]$, and are shown in Table 1. 


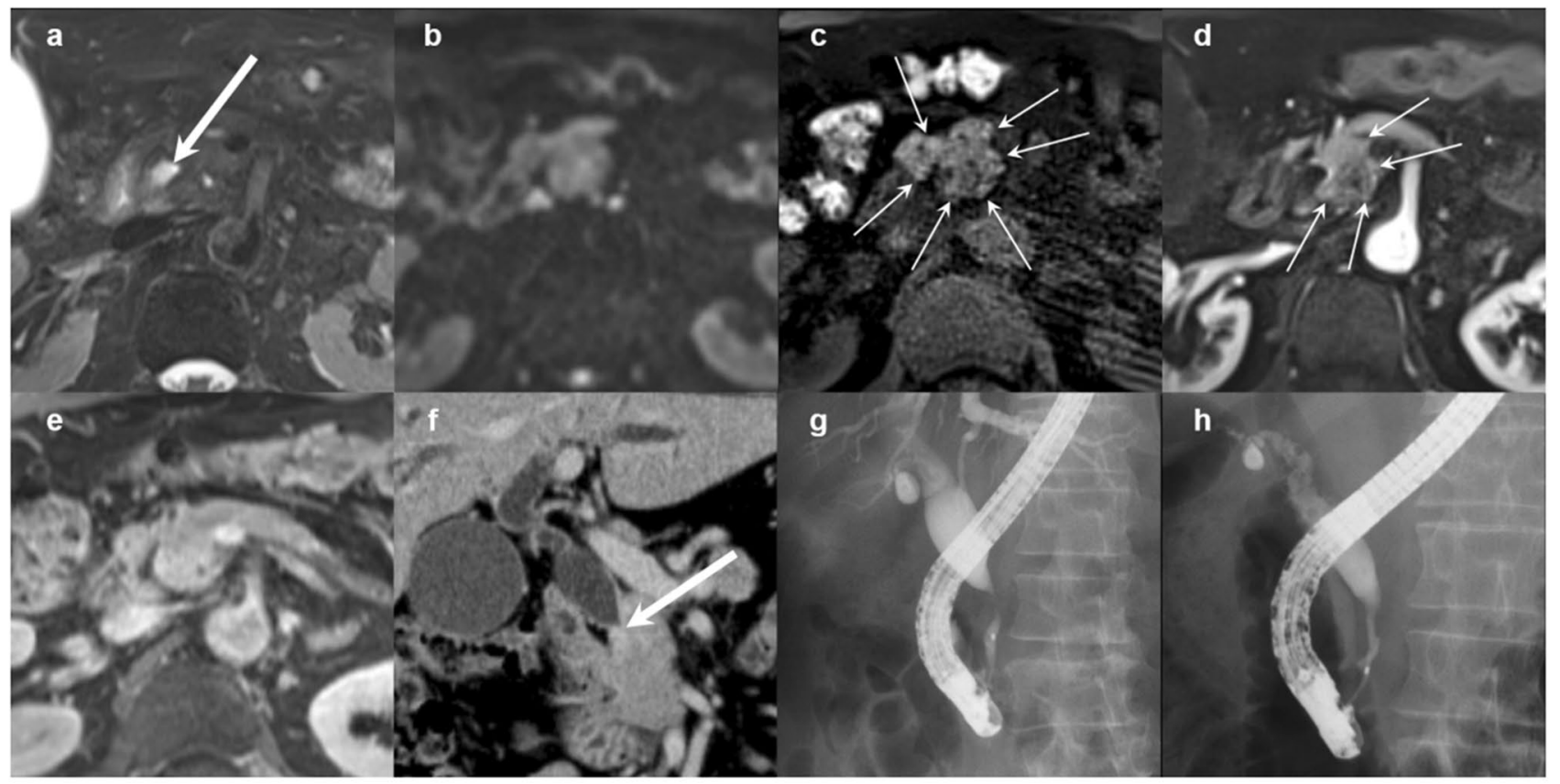

i Radiologic findings

\begin{tabular}{lc}
\hline Pancreatic swelling & - \\
\hline Speckled hyperintensity on FS-T1WI & $\bigcirc$ \\
\hline Speckled enhancement on pancreatic phase & $\bigcirc$ \\
\hline Capsule-like rim & - \\
\hline Homogeneous delayed enhancement & $\bigcirc$ \\
\hline Duct-penetrating sign & - \\
\hline Enhanced duct sign & - \\
\hline No MPD dilatation & - \\
\hline Extra-pancreatic lesions & $\bigcirc$ \\
\hline
\end{tabular}

Fig. 21 Focal autoimmune pancreatitis (AIP) with dilatation of the common bile duct (CBD). a A fat-suppressed T2-weighted spinecho image shows mild dilatation of the CBD (arrow). b A diffusionweighted image $(b=1000)$ shows a hyperintense area in the pancreatic head, suggesting focal AIP. c A fat-suppressed T1-weighted spin-echo image shows speckled hyperintensity in the lesion in the pancreatic head. d A pancreatic-phase dynamic contrast-enhanced magnetic resonance (DCE-MR) image shows speckled enhancement

\subsection{Atypical findings of AIP}

Focal AIP should be differentiated from other hypovascular lesions.

Calcifications and cystic lesions may be seen.

\subsubsection{Description}

The frequency of focal AIP varies, and it is sometimes difficult to differentiate focal AIP from other hypovascular tumors such as pancreatic cancer (acinar cell carcinoma within the lesion. e A delayed-phase DCE-MR image shows homogeneous delayed enhancement of the lesion. $\mathbf{f}$ A coronal reconstructed image of the DCE-CT pancreatic phase shows slight wall thickening and dilatation of the CBD with a beak-like stenosis (arrow). $\mathbf{g}$ An ERCP image shows beak-like stenosis and upstream dilatation of the CBD. h An ERCP image after steroid treatment shows improvement of the CBD stenosis

with degeneration or necrosis), neuroendocrine tumor (with degeneration), malignant lymphoma, and metastatic tumor.

If calcification is seen in the pancreas, chronic pancreatitis should be considered as one of the differential diagnoses. It should be noted that AIP sometimes resembles chronic pancreatitis in patients with pancreatic head swelling, no narrowing of the MPD in the pancreatic body, and repeated relapses, because calcification in the pancreas is seen in $7-19.6 \%$ of these patients (Fig. 18) [44-46]. Many cases with AIP associated with cystic lesions have been reported 


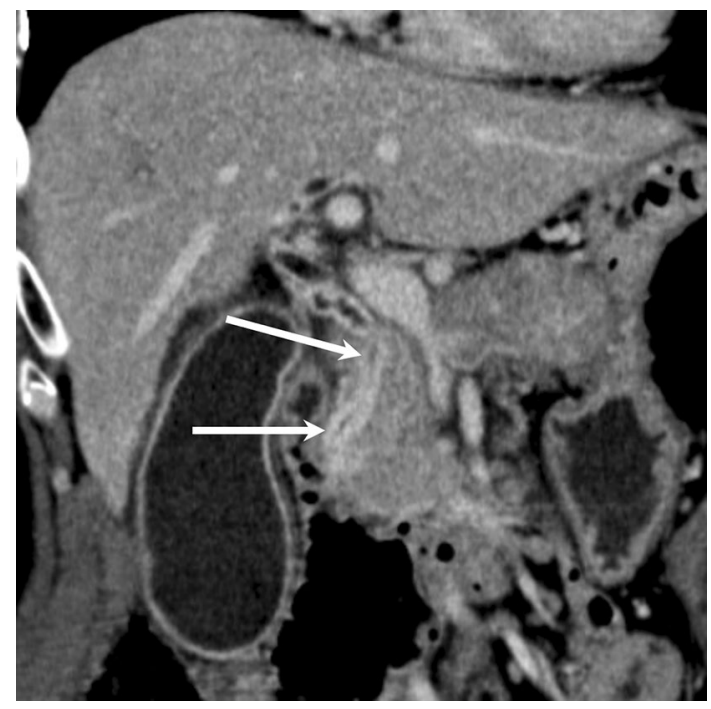

Fig. 22 Autoimmune pancreatitis (AIP) with bile duct wall thickening. A portal-phase dynamic contrast-enhanced computed tomography image shows diffuse pancreatic swelling and bile duct wall thickening with enhancement (arrows). Dilatation of the intrahepatic bile ducts is not seen

and most of the lesions improved or disappeared upon corticosteroid therapy [47-49] (Fig. 19).

\section{Diseases requiring differentiation from AIP}

\subsection{Differential diagnosis}

When diffuse pancreatic swelling is seen, diffuse pancreatic cancer, malignant lymphoma, sarcoidosis, and chronic pancreatitis should be considered.

Differential diagnosis for a focal hypovascular lesion is described in Section 2.11.

\subsubsection{Description}

As described above, differential diagnosis after considering whether the lesion is diffuse or focal is a key to differentiation. It is also important to differentiate AIP from other malignant tumors, especially pancreatic cancer and malignant lymphoma. If the lesion is difficult to differentiate, EUSguided fine-needle aspiration should be considered. Although diagnostic therapy by corticosteroid administration is one of the options, it should be noted that the lesion can rapidly progress and the timing of surgical treatment may be missed.

\subsection{Pancreatic cancer}

It is necessary to make a comprehensive diagnosis for differentiation of AIP from pancreatic cancer because the sensitivity and specificity of the useful findings vary (Table 2).

The pancreatic duct within the lesion and the bile duct and vessels should be paid attention for accurate diagnosis.

When differentiation is difficult, pathological diagnosis by EUS-fine-needle aspiration should be considered.

\subsubsection{Description}

Specific CT and MR findings for AIP, such as the capsulelike rim, duct-penetrating sign, speckled/dotted enhancement, and homogeneous delayed enhancement, are adopted as useful findings for differentiation of AIP from pancreatic cancer in the JPS2018. However, the capsule-like rim and duct-penetration sign are rarely seen in small lesions. Although arterial wall irregularity is sometimes seen in AIP (Fig. 20), severe arterial stenosis around the lesion suggests a high possibility of malignancy (pancreatic cancer). Stenosis of the splenic vein and bile duct dilatation due to the lesion are seen in both AIP and pancreatic cancer, and these findings are not useful for differentiation (Figs. 20, 21). Bile duct wall thickening without stenosis is sometimes seen in AIP (Fig. 22). On DWI, the ADC value of AIP is lower than that of pancreatic cancer [16-19], but it is difficult to differentiate between pancreatic cancer and pancreatitis accompanying the tumor. However, this finding is not clinically useful for differentiation, because the cut-off values vary and depend on the MR units and scan parameters used. On FDG-PET, diffuse or multifocal accumulation in the pancreatic lesion and accumulation in extrapancreatic lesions are highly suggestive of AIP [38, 50]. Improvement of pancreas swelling and decreased accumulation after steroid therapy are also suggestive of AIP [40, 51]. It should be noted that pathological diagnosis by EUS-fine needle aspiration should be considered if any radiologic findings suggestive of pancreatic cancer are seen (Fig. 23).

\subsection{Malignant lymphoma}

Malignant lymphoma can show the same imaging findings as AIP.

\subsubsection{Description}

Various types of imaging findings are seen in malignant lymphoma of the pancreas, including primary and secondary lesions. Well-circumscribed nodular type with solitary pancreatic mass, diffuse type with pancreatic enlargement (Fig. 24), peripheral lymphomatous involvement mimicking autoimmune pancreatitis, multi-nodular type, and invasion from adjacent peri-pancreatic lymphomatous lesion have been reported [52]. CT or MR findings for a lesion with diffuse pancreatic involvement are sometimes similar to those for 


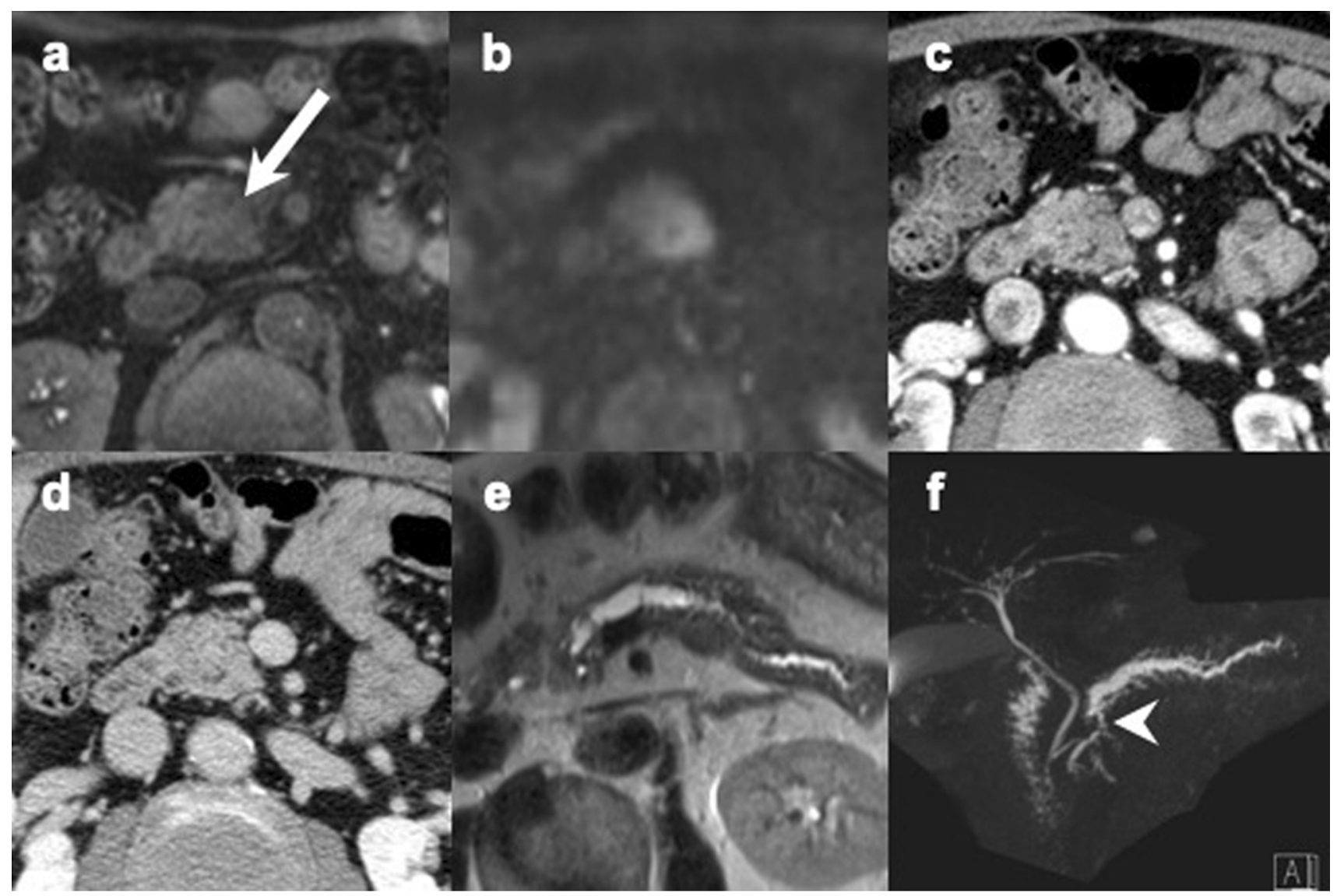

g Radiologic findings

\begin{tabular}{lc}
\hline Pancreatic swelling & - \\
\hline Speckled hyperintensity on FS-T1WI & $\bigcirc$ \\
\hline Speckled enhancement on pancreatic phase & - \\
\hline Capsule-like rim & - \\
\hline Homogeneous delayed enhancement & $\bigcirc$ \\
\hline Duct-penetrating sign & $\bigcirc$ \\
\hline Enhanced duct sign & - \\
\hline No MPD dilatation & - \\
\hline Extra-pancreatic lesions & - \\
\hline
\end{tabular}

Fig. 23 Focal autoimmune pancreatitis (AIP) with dilatation of the main pancreatic duct (MPD). a A fat-suppressed T1-weighted image shows speckled hyperintensity in focal AIP of the pancreatic head (arrow). b A diffusion-weighted image $(b=1000)$ shows the lesion as a hyperintense area. c A pancreatic-phase dynamic contrast-enhanced computed tomography (DCE-CT) image shows slight enhancement of the lesion. d A delayed-phase DCE-CT image shows homogeneous delayed enhancement of the lesion. e, f An ultrafast T2-weighted spin-echo image (e) and a magnetic resonance cholangiopancreatography image (f) show the duct-penetrating sign (arrowhead), but the upstream MPD is dilated. This case was pathologically analyzed and diagnosed as AIP 


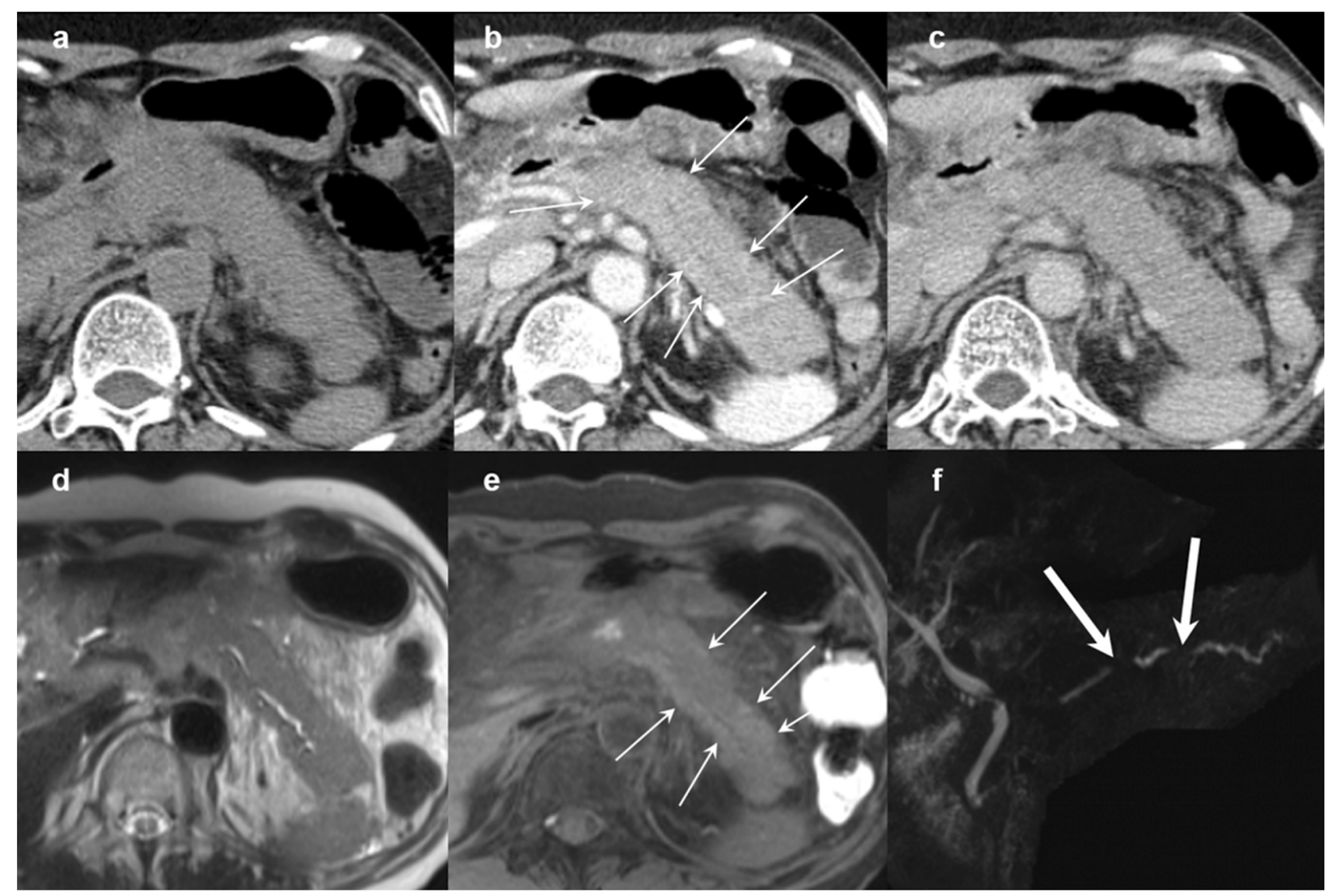

g

\begin{tabular}{lc}
\hline Radiologic findings & \\
\hline Pancreatic swelling & $\bigcirc$ \\
\hline Speckled hyperintensity on FS-T1WI & $\bigcirc$ \\
\hline Speckled enhancement on pancreatic phase & $\bigcirc$ \\
\hline Capsule-like rim & - \\
\hline Homogeneous delayed enhancement & $\bigcirc$ \\
\hline Duct-penetrating sign & $\bigcirc$ \\
\hline Enhanced duct sign & - \\
\hline No MPD dilatation & $\bigcirc$ \\
\hline Extra-pancreatic lesions & - \\
\hline
\end{tabular}

Fig. 24 Pancreatic malignant diffuse large B cell lymphoma with diffuse pancreatic enlargement. a A non-contrast computed tomography (CT) image shows diffuse swelling of the pancreas. b A pancreaticphase dynamic contrast-enhanced computed tomography (DCE-CT) image shows speckled/dotted enhancement of the pancreas (small arrows). The main pancreatic duct (MPD) is present within the pancreatic lesion and no upstream dilatation is seen. c A delayed-phase DCE-CT image shows homogeneous delayed enhancement. A cap- sule-like rim is unclear. d An ultrafast T2-weighted spin-echo image shows diffuse pancreatic enlargement with a slight increase in the signal. Irregular narrowing of the MPD with no upstream dilatation is seen. e A fat-suppressed T1-weighted spin-echo image shows speckled/dotted hyperintense areas (small arrows). f A magnetic resonance cholangiopancreatography image shows skipped narrowing of the MPD (arrows) 
AIP. Presence of lymphadenopathy below the level of the left renal vein is highly suggestive of pancreatic lymphoma [53].

\subsection{Chronic pancreatitis}

Pancreatic atrophy and calcification are sometimes seen in AIP.

\subsubsection{Description}

Calcification is sometimes seen in AIP patients with repeated relapses (Fig. 18). Risk factors for transformation of AIP into chronic pancreatitis are pancreatic head swelling, no MPD narrowing in the pancreatic body, and repeated relapses [45]. Therefore, AIP should be considered if chronic pancreatitis is seen without a history of drinking.

Acknowledgements The authors thank Alison Sherwin, $\mathrm{PhD}$, from Edanz Group (https://en-author-services.edanzgroup.com/) for editing a draft of this manuscript.

Funding This study was partly supported by Health and Labor Sciences Research Grants for The Research Program on Intractable Diseases from the Ministry of Labor and Welfare of Japan.

\section{Compliance with ethical standards}

Conflict of interest The authors declare that they have no conflict of interest.

Ethical statement This article does not contain any studies with human participants or animals performed by any of the authors.

\section{References}

1. Kawa S, Kamisawa T, Notohara K, Fujinaga Y, Inoue D, Koyama $\mathrm{T}$, et al. Japanese clinical diagnostic criteria for autoimmune pancreatitis, 2018: revision of Japanese clinical diagnostic criteria for autoimmune pancreatitis, 2011. Pancreas. 2020;49(1):e13-e1414.

2. Kamisawa T, Chen PY, Tu Y, Nakajima H, Egawa N, Tsuruta K, et al. MRCP and MRI findings in 9 patients with autoimmune pancreatitis. World J Gastroenterol. 2006;12(18):2919-22.

3. Frulloni L, Scattolini C, Falconi M, Zamboni G, Capelli P, Manfredi R, et al. Autoimmune pancreatitis: differences between the focal and diffuse forms in 87 patients. Am J Gastroenterol. 2009;104(9):2288-94.

4. Negrelli R, Manfredi R, Pedrinolla B, Boninsegna E, Ventriglia A, Mehrabi S, et al. Pancreatic duct abnormalities in focal autoimmune pancreatitis: MR/MRCP imaging findings. Eur Radiol. 2015;25(2):359-67.

5. Okazaki K, Kawa S, Kamisawa T, Ito T, Inui K, Irie H, et al. Amendment of the Japanese consensus guidelines for autoimmune pancreatitis, 2013 I. Concept and diagnosis of autoimmune pancreatitis. J Gastroenterol. 2014;49(4):567-88.

6. Kawa S, Okazaki K, Kamisawa T, Kubo K, Ohara H, Hasebe O, et al. Amendment of the Japanese consensus guidelines for autoimmune pancreatitis, 2013 II. Extrapancreatic lesions, differential diagnosis. J Gastroenterol. 2014;49(5):765-84.
7. Kamisawa T, Okazaki K, Kawa S, Ito T, Inui K, Irie H, et al. Amendment of the Japanese consensus guidelines for autoimmune pancreatitis, 2013 III. Treatment and prognosis of autoimmune pancreatitis. J Gastroenterol. 2014;49(6):961-70.

8. Takahashi N, Fletcher JG, Fidler JL, Hough DM, Kawashima A, Chari ST. Dual-phase CT of autoimmune pancreatitis: a multireader study. AJR Am J Roentgenol. 2008;190(2):280-6.

9. Kamisawa T, Tu Y, Egawa N, Tsuruta K, Okamoto A, Kodama M, et al. Can MRCP replace ERCP for the diagnosis of autoimmune pancreatitis? Abdom Imaging. 2009;34(3):381-4.

10. Park SH, Kim MH, Kim SY, Kim HJ, Moon SH, Lee SS, et al. Magnetic resonance cholangiopancreatography for the diagnostic evaluation of autoimmune pancreatitis. Pancreas. 2010;39(8):1191-8.

11. Yanagisawa S, Fujinaga $Y$, Watanabe T, Maruyama M, Muraki T, Takahashi M, et al. Usefulness of three-dimensional magnetic resonance cholangiopancreatography with partial maximum intensity projection for diagnosing autoimmune pancreatitis. Pancreatology. 2017;17(4):567-71.

12. Motosugi U, Ichikawa T, Morisaka H, Sou H, Muhi A, Kimura K, et al. Detection of pancreatic carcinoma and liver metastases with gadoxetic acid-enhanced MR imaging: comparison with contrastenhanced multi-detector row CT. Radiology. 2011;260(2):446-53.

13. Chandan VS, Iacobuzio-Donahue C, Abraham SC. Patchy distribution of pathologic abnormalities in autoimmune pancreatitis: implications for preoperative diagnosis. Am J Surg Pathol. 2008;32(12):1762-9.

14. Fujinaga $\mathrm{Y}$, Kadoya M, Hamano H, Kawa S, Momose M, Kawakami S, et al. Radiologic findings of IgG4-related disease. Curr Immunol Rev. 2011;7(2):186-203.

15. Sugiyama Y, Fujinaga Y, Kadoya M, Ueda K, Kurozumi M, Hamano $\mathrm{H}$, et al. Characteristic magnetic resonance features of focal autoimmune pancreatitis useful for differentiation from pancreatic cancer. Jpn J Radiol. 2012;30(4):296-309.

16. Muhi A, Ichikawa T, Motosugi U, Sou H, Sano K, Tsukamoto $\mathrm{T}$, et al. Mass-forming autoimmune pancreatitis and pancreatic carcinoma: differential diagnosis on the basis of computed tomography and magnetic resonance cholangiopancreatography, and diffusion-weighted imaging findings. J Magn Reson Imaging. 2012;35(4):827-36.

17. Hur BY, Lee JM, Lee JE, Park JY, Kim SJ, Joo I, et al. Magnetic resonance imaging findings of the mass-forming type of autoimmune pancreatitis: comparison with pancreatic adenocarcinoma. J Magn Reson Imaging. 2012;36(1):188-97.

18. Choi SY, Kim SH, Kang TW, Song KD, Park HJ, Choi YH. Differentiating mass-forming autoimmune pancreatitis from pancreatic ductal adenocarcinoma on the basis of contrast-enhanced MRI and DWI findings. AJR Am J Roentgenol. 2016;206(2):291-300.

19. Kamisawa T, Takuma K, Anjiki H, Egawa N, Hata T, Kurata $\mathrm{M}$, et al. Differentiation of autoimmune pancreatitis from pancreatic cancer by diffusion-weighted MRI. Am J Gastroenterol. 2010;105(8):1870-5.

20. Haaga JR, Alfidi RJ, Zelch MG, Meany TF, Boller M, Gonzalez L, et al. Computed tomography of the pancreas. Radiology. 1976;120(3):589-95.

21. Furuhashi N, Suzuki K, Sakurai Y, Ikeda M, Kawai Y, Naganawa S. Differentiation of focal-type autoimmune pancreatitis from pancreatic carcinoma: assessment by multiphase contrast-enhanced CT. Eur Radiol. 2015;25(5):1366-74.

22. Kwon JH, Kim JH, Kim SY, Byun JH, Kim HJ, Lee MG, et al. Differentiating focal autoimmune pancreatitis and pancreatic ductal adenocarcinoma: contrast-enhanced MRI with special emphasis on the arterial phase. Eur Radiol. 2019;29(11):5763-71.

23. Irie H, Honda H, Baba S, Kuroiwa T, Yoshimitsu K, Tajima T, et al. Autoimmune pancreatitis: CT and MR characteristics. AJR Am J Roentgenol. 1998;170(5):1323-7. 
24. Kawamoto S, Siegelman SS, Hruban RH, Fishman EK. Lymphoplasmacytic sclerosing pancreatitis (autoimmune pancreatitis): evaluation with multidetector CT. Radiographics. 2008;28(1):157-70.

25. Sahani DV, Kalva SP, Farrell J, Maher MM, Saini S, Mueller PR, et al. Autoimmune pancreatitis: imaging features. Radiology. 2004;233(2):345-52.

26. Sun GF, Zuo CJ, Shao CW, Wang JH, Zhang J. Focal autoimmune pancreatitis: radiological characteristics help to distinguish from pancreatic cancer. World J Gastroenterol. 2013;19(23):3634-41.

27. Kim HJ, Kim YK, Jeong WK, Lee WJ, Choi D. Pancreatic duct "Icicle sign" on MRI for distinguishing autoimmune pancreatitis from pancreatic ductal adenocarcinoma in the proximal pancreas. Eur Radiol. 2015;25(6):1551-600.

28. Lee S, Kim JH, Kim SY, Byun JH, Kim HJ, Kim MH, et al. Comparison of diagnostic performance between CT and MRI in differentiating non-diffuse-type autoimmune pancreatitis from pancreatic ductal adenocarcinoma. Eur Radiol. 2018;28(12):5267-74.

29. Wakabayashi T, Kawaura Y, Satomura Y, Watanabe H, Motoo Y, Okai T, et al. Clinical and imaging features of autoimmune pancreatitis with focal pancreatic swelling or mass formation: comparison with so-called tumor-forming pancreatitis and pancreatic carcinoma. Am J Gastroenterol. 2003;98(12):2679-87.

30. Ichikawa T, Sou H, Araki T, Arbab AS, Yoshikawa T, Ishigame K, et al. Duct-penetrating sign at MRCP: usefulness for differentiating inflammatory pancreatic mass from pancreatic carcinomas. Radiology. 2001;221(1):107-16.

31. Naitoh I, Nakazawa T, Hayashi K, Okumura F, Miyabe K, Shimizu $\mathrm{S}$, et al. Clinical differences between mass-forming autoimmune pancreatitis and pancreatic cancer. Scand J Gastroenterol. 2012;47(5):607-13.

32. Tokoro H, Yamada A, Suzuki T, Kito Y, Adachi Y, Hayashihara H, et al. Usefulness of breath-hold compressed sensing accelerated three-dimensional magnetic resonance cholangiopancreatography (MRCP) added to respiratory-gating conventional MRCP. Eur J Radiol. 2020;122:108765.

33. Tamura R, Ishibashi T, Takahashi S. Chronic pancreatitis: MRCP versus ERCP for quantitative caliber measurement and qualitative evaluation. Radiology. 2006;238(3):920-8.

34. Kawai Y, Suzuki K, Itoh S, Takada A, Mori Y, Naganawa S. Autoimmune pancreatitis: assessment of the enhanced duct sign on multiphase contrast-enhanced computed tomography. Eur J Radiol. 2012;81(11):3055-60.

35. Nishino T, Oyama H, Toki F, Shiratori K. Differentiation between autoimmune pancreatitis and pancreatic carcinoma based on endoscopic retrograde cholangiopancreatography findings. J Gastroenterol. 2010;45(9):988-96.

36. Nakajo M, Jinnouchi S, Fukukura Y, Tanabe H, Tateno R, Nakajo M. The efficacy of whole-body FDG-PET or PET/CT for autoimmune pancreatitis and associated extrapancreatic autoimmune lesions. Eur J Nucl Med Mol Imaging. 2007;34(12):2088-95.

37. Ishii S, Shishido F, Miyajima M, Sakuma K, Shigihara T, Kikuchi $\mathrm{K}$. Whole-body gallium-67 scintigraphic findings in IgG4-related disease. Clin Nucl Med. 2011;36(7):542-5.

38. Ozaki Y, Oguchi K, Hamano H, Arakura N, Muraki T, Kiyosawa $\mathrm{K}$, et al. Differentiation of autoimmune pancreatitis from suspected pancreatic cancer by fluorine- 18 fluorodeoxyglucose positron emission tomography. J Gastroenterol. 2008;43(2):144-51.

39. Lee TY, Kim MH, Park DH, Seo DW, Lee SK, Kim JS, et al. Utility of 18F-FDG PET/CT for differentiation of autoimmune pancreatitis with atypical pancreatic imaging findings from pancreatic cancer. AJR Am J Roentgenol. 2009;193(2):343-8.

40. Matsubayashi H, Furukawa H, Maeda A, Matsunaga K, Kanemoto $\mathrm{H}$, Uesaka $\mathrm{K}$, et al. Usefulness of positron emission tomography in the evaluation of distribution and activity of systemic lesions associated with autoimmune pancreatitis. Pancreatology. 2009;9(5):694-9.

41. Shimosegawa T, Chari ST, Frulloni L, Kamisawa T, Kawa S, Mino-Kenudson M, et al. International consensus diagnostic criteria for autoimmune pancreatitis: guidelines of the International Association of Pancreatology. Pancreas. 2011;40(3):352-8.

42. Ohara H, Nakazawa T, Sano H, Ando T, Okamoto T, Takada H, et al. Systemic extrapancreatic lesions associated with autoimmune pancreatitis. Pancreas. 2005;31(3):232-7.

43. Fujinaga Y, Kadoya M, Kawa S, Hamano H, Ueda K, Momose $\mathrm{M}$, et al. Characteristic findings in images of extra-pancreatic lesions associated with autoimmune pancreatitis. Eur J Radiol. 2010;76(2):228-38.

44. Takayama M, Hamano H, Ochi Y, Saegusa H, Komatsu K, Muraki T, et al. Recurrent attacks of autoimmune pancreatitis result in pancreatic stone formation. Am J Gastroenterol. 2004;99(5):932-7.

45. Maruyama M, Arakura N, Ozaki Y, Watanabe T, Ito T, Yoneda S, et al. Type 1 autoimmune pancreatitis can transform into chronic pancreatitis: a long-term follow-up study of 73 Japanese patients. Int J Rheumatol. 2013;2013:272595.

46. Ito T, Kawa S, Matsumoto A, Kubota K, Kamisawa T, Okazaki K, et al. Risk factors for pancreatic stone formation in type 1 autoimmune pancreatitis: a long-term Japanese multicenter analysis of 624 patients. Pancreas. 2019;48(1):49-544.

47. Kuraishi Y, Watanabe T, Muraki T, Ashihara N, Ozawa M, Nakamura A, et al. Effectiveness of steroid therapy for pancreatic cysts complicating autoimmune pancreatitis and management strategy for cyst-related complications. Scand J Gastroenterol. 2019;54(6):773-9.

48. Muraki T, Hamano H, Ochi Y, Arakura N, Takayama M, Komatsu $\mathrm{K}$, et al. Corticosteroid-responsive pancreatic cyst found in autoimmune pancreatitis. J Gastroenterol. 2005;40(7):761-6.

49. Nishimura N, Tamada K, Wada S, Ohashi A, Hatanaka H, Nakazawa K, et al. Autoimmune pancreatitis associated with a large pancreatic pseudocyst that disappeared after corticosteroid therapy: a case report and literature review. Clin J Gastroenterol. 2009;2(3):199-203.

50. Lee NK, Kim S, Kim DU, Seo HI, Kim HS, Jo HJ, et al. Diffusion-weighted magnetic resonance imaging for non-neoplastic conditions in the hepatobiliary and pancreatic regions: pearls and potential pitfalls in imaging interpretation. Abdom Imaging. 2015;40(3):643-62.

51. Shigekawa M, Yamao K, Sawaki A, Hara K, Takagi T, Bhatia V, et al. Is (18)F-fluorodeoxyglucose positron emission tomography meaningful for estimating the efficacy of corticosteroid therapy in patients with autoimmune pancreatitis? J Hepatobiliary Pancreat Sci. 2010;17(3):269-74.

52. Fujinaga Y, Lall C, Patel A, Matsushita T, Sanyal R, Kadoya M. MR features of primary and secondary malignant lymphoma of the pancreas: a pictorial review. Insights Imaging. 2013;4(3):321-9.

53. Merkle EM, Bender GN, Brambs HJ. Imaging findings in pancreatic lymphoma: differential aspects. AJR Am J Roentgenol. 2000;174(3):671-5.

54. Kamisawa T, Funata N, Hayashi Y, Tsuruta K, Okamoto A, Amemiya K, et al. Close relationship between autoimmune pancreatitis and multifocal fibrosclerosis. Gut. 2003;52(5):683-7.

55. van der Vliet HJ, Perenboom RM. Multiple pseudotumors in IgG4-associated multifocal systemic fibrosis. Ann Intern Med. 2004;141(11):896-7.

56. Chan SK, Cheuk W, Chan KT, Chan JK. IgG4-related sclerosing pachymeningitis: a previously unrecognized form of central nervous system involvement in IgG4-related sclerosing disease. Am J Surg Pathol. 2009;33(8):1249-52. 
57. Saegusa H, Momose M, Kawa S, Hamano H, Ochi Y, Takayama $\mathrm{M}$, et al. Hilar and pancreatic gallium-67 accumulation is characteristic feature of autoimmune pancreatitis. Pancreas. 2003;27(1):20-5.

58. Taniguchi T, Ko M, Seko S, Nishida O, Inoue F, Kobayashi H, et al. Interstitial pneumonia associated with autoimmune pancreatitis. Gut. 2004;53(5):770 (author reply -1).

59. Hirano K, Kawabe T, Komatsu Y, Matsubara S, Togawa O, Arizumi $\mathrm{T}$, et al. High-rate pulmonary involvement in autoimmune pancreatitis. Intern Med J. 2006;36(1):58-61.

60. Sugimoto T, Tanaka Y, Morita Y, Kume S, Uzu T, Kashiwagi A. Is tubulointerstitial nephritis and uveitis syndrome associated with IgG4-related systemic disease? Nephrology (Carlton). 2008;13(1):89.

61. Nakazawa T, Ohara H, Yamada T, Ando H, Sano H, Kajino $\mathrm{S}$, et al. Atypical primary sclerosing cholangitis cases associated with unusual pancreatitis. Hepatogastroenterology. 2001;48(39):625-30.

62. Komatsu K, Hamano H, Ochi Y, Takayama M, Muraki T, Yoshizawa K, et al. High prevalence of hypothyroidism in patients with autoimmune pancreatitis. Dig Dis Sci. 2005;50(6):1052-7.

63. Watanabe T, Maruyama M, Ito T, Fujinaga Y, Ozaki Y, Maruyama $\mathrm{M}$, et al. Clinical features of a new disease concept, IgG4-related thyroiditis. Scand J Rheumatol. 2013;42(4):325-30.

64. Hamano H, Kawa S, Ochi Y, Unno H, Shiba N, Wajiki M, et al. Hydronephrosis associated with retroperitoneal fibrosis and sclerosing pancreatitis. Lancet. 2002;359(9315):1403-4.

65. Zen Y, Kasahara Y, Horita K, Miyayama S, Miura S, Kitagawa $\mathrm{S}$, et al. Inflammatory pseudotumor of the breast in a patient with a high serum IgG4 level: histologic similarity to sclerosing pancreatitis. Am J Surg Pathol. 2005;29(2):275-8.

66. Zen Y, Inoue D, Kitao A, Onodera M, Abo H, Miyayama S, et al. IgG4-related lung and pleural disease: a clinicopathologic study of 21 cases. Am J Surg Pathol. 2009;33(12):1886-933.

67. Zen Y, Fujii T, Sato Y, Masuda S, Nakanuma Y. Pathological classification of hepatic inflammatory pseudotumor with respect to IgG4-related disease. Mod Pathol. 2007;20(8):884-94.

68. Takeda S, Haratake J, Kasai T, Takaeda C, Takazakura E. IgG4-associated idiopathic tubulointerstitial nephritis complicating autoimmune pancreatitis. Nephrol Dial Transplant. 2004;19(2):474-6.

69. Uchiyama-Tanaka Y, Mori Y, Kimura T, Sonomura K, Umemura $\mathrm{S}$, Kishimoto N, et al. Acute tubulointerstitial nephritis associated with autoimmune-related pancreatitis. Am J Kidney Dis. 2004;43(3):e18-25.
70. Shinji A, Sano K, Hamano H, Unno H, Fukushima M, Nakamura N, et al. Autoimmune pancreatitis is closely associated with gastric ulcer presenting with abundant IgG4-bearing plasma cell infiltration. Gastrointest Endosc. 2004;59(4):506-11.

71. Kasashima S, Zen Y, Kawashima A, Konishi K, Sasaki H, Endo M, et al. Inflammatory abdominal aortic aneurysm: close relationship to IgG4-related periaortitis. Am J Surg Pathol. 2008;32(2):197-204.

72. Mizushima I, Kasashima S, Fujinaga Y, Notohara K, Saeki T, Zen Y, et al. Clinical and pathological characteristics of IgG4-related periaortitis/periarteritis and retroperitoneal fibrosis diagnosed based on experts' diagnosis. Ann Vasc Dis. 2019;12(4):460-72.

73. Unno H, Saegusa H, Fukushima M, Hamano H. Usefulness of endoscopic observation of the main duodenal papilla in the diagnosis of sclerosing pancreatitis. Gastrointest Endosc. 2002;56(6):880-4.

74. Umemura T, Zen Y, Hamano H, Kawa S, Nakanuma Y, Kiyosawa K. Immunoglobin G4-hepatopathy: association of immunoglobin G4-bearing plasma cells in liver with autoimmune pancreatitis. Hepatology. 2007;46(2):463-71.

75. Umemura T, Zen Y, Hamano H, Joshita S, Ichijo T, Yoshizawa $\mathrm{K}$, et al. Clinical significance of immunoglobulin G4-associated autoimmune hepatitis. J Gastroenterol. 2011;46(Suppl 1):48-55.

76. Yoshimura Y, Takeda S, Ieki Y, Takazakura E, Koizumi H, Takagawa $\mathrm{K}$. IgG4-associated prostatitis complicating autoimmune pancreatitis. Intern Med. 2006;45(15):897-901.

77. Watanabe T, Fujinaga Y, Kawakami S, Hatta T, Hamano H, Kawa $\mathrm{S}$, et al. Infraorbital nerve swelling associated with autoimmune pancreatitis. Jpn J Radiol. 2011;29(3):194-201.

78. Inoue $\mathrm{D}$, Zen $\mathrm{Y}$, Sato $\mathrm{Y}$, Abo $\mathrm{H}$, Demachi $\mathrm{H}$, Uchiyama A, et al. IgG4-related perineural disease. Int J Rheumatol. 2012;2012:401890.

79. Nakamura A, Funatomi H, Katagiri A, Katayose K, Kitamura $\mathrm{K}$, Seki T, et al. A case of autoimmune pancreatitis complicated with immune thrombocytopenia during maintenance therapy with prednisolone. Dig Dis Sci. 2003;48(10):1968-71.

Publisher's Note Springer Nature remains neutral with regard to jurisdictional claims in published maps and institutional affiliations. 